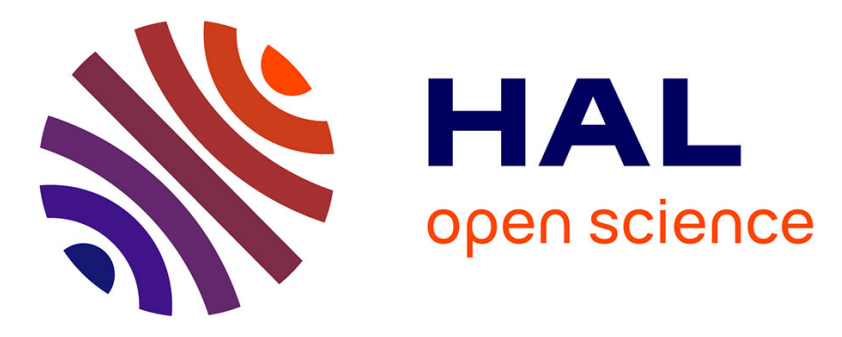

\title{
The Calern Asteroid Polarimetric Survey using the Torino polarimeter: assessment of instrument performances and first scientific results
}

M. Devogèle, A. Cellino, S. Bagnulo, J. Rivet, P. Bendjoya, L. Abe, C. Pernechele, G. Massone, D. Vernet, Paolo Tanga, et al.

\section{To cite this version:}

M. Devogèle, A. Cellino, S. Bagnulo, J. Rivet, P. Bendjoya, et al.. The Calern Asteroid Polarimetric Survey using the Torino polarimeter: assessment of instrument performances and first scientific results. Monthly Notices of the Royal Astronomical Society, 2016, 465 (4), pp.4335-4347. 10.1093/mnras/stw2952 . hal-03526061

\section{HAL Id: hal-03526061 https://hal.science/hal-03526061}

Submitted on 14 Jan 2022

HAL is a multi-disciplinary open access archive for the deposit and dissemination of scientific research documents, whether they are published or not. The documents may come from teaching and research institutions in France or abroad, or from public or private research centers.
L'archive ouverte pluridisciplinaire HAL, est destinée au dépôt et à la diffusion de documents scientifiques de niveau recherche, publiés ou non, émanant des établissements d'enseignement et de recherche français ou étrangers, des laboratoires publics ou privés. 


\title{
The Calern Asteroid Polarimetric Survey using the Torino polarimeter: assessment of instrument performances and first scientific results
}

\author{
M. Devogèle,${ }^{1,2 \star}$ A. Cellino, ${ }^{3}$ S. Bagnulo, ${ }^{4}$ J. P. Rivet, ${ }^{2}$ P. Bendjoya, ${ }^{2}$ L. Abe,${ }^{2}$ \\ C. Pernechele, ${ }^{5}$ G. Massone, ${ }^{6}$ D. Vernet, ${ }^{2}$ P. Tanga ${ }^{2}$ and C. Dimur ${ }^{2}$ \\ ${ }^{1}$ Université de Liège, Space Sciences, Technologies and Astrophysics Research (STAR) Institute, Allée du 6 Août $19 \mathrm{c}$, Sart Tilman, 4000 Liège, Belgium \\ ${ }^{2}$ Laboratoire Lagrange, UMR7293, Univ. Côte d'Azur, CNRS, Obs. de la Côte d'Azur, Bv de l'Observatoire, S 34229,06304 Nice, France \\ ${ }^{3}$ INAF - Osservatorio Astrofisico di Torino, Pino Torinese, Italy \\ ${ }^{4}$ Armagh Observatory and Planetarium, College Hill, Armagh BT61 9DG \\ ${ }^{5}$ INAF - Osservatorio Astronomico di Padova, Padova, Italy \\ ${ }^{6}$ INAF - Osservatorio Astrofisico di Torino, Pino Torinese, Italy
}

Accepted 2016 November 14. Received 2016 November 7; in original form 2016 March 23

\begin{abstract}
A new polarimeter based on the wedged double Wollaston concept has been built at the Torino Observatory and installed on a 1-m telescope at the Calern observing station of the Observatoire de la Côte d'Azur, France. Its main purpose is to carry out a polarimetric survey of minor Solar system objects, which is called the Calern Asteroid Polarimetric Survey. In this paper, we describe the new Torino polarimeter and the results of preliminary scientific validation tests. A number of standard stars with known polarization states, as well as a number of asteroids for which the polarimetric properties are known, have been observed in order to assess the instrument's accuracy. The instrumental polarization has been found to be stable within a few $10^{-4}$ units. A total of 124 new polarimetric observation of 78 asteroids are presented. In the case of asteroids already observed in the past, the new data are in agreement with available phase-polarization curves with error bars smaller than most previously published data. We also present data for 21 asteroids that have never been observed before in polarimetry.
\end{abstract}

Key words: polarization-instrumentation: polarimeters - minor planets, asteroids: general.

\section{INTRODUCTION}

Polarimetry is an important tool to obtain a physical characterization of small Solar system bodies. The change of linear polarization of scattered sunlight in different illumination conditions can provide information about some properties of the layer of regolith covering the surfaces of atmosphereless Solar system bodies (Petrova, Tishkovets \& Jockers 2004). In particular, polarimetry is a primary technique to determine the geometric albedo of the surfaces (Cellino et al. 2015b). Recent review papers of this subject have been published by Cellino, Gil-Hutton \& Belskaya (2015a) and Belskaya et al. (2015), where interested readers will also find updated lists of bibliographic references. An analysis of the dependence of linear polarization upon wavelength has been published by Bagnulo, Cellino \& Sterzik (2015).

The primary goal of classical asteroid polarimetry is to determine the so-called phase-polarization curve. This curve describes the change of the fraction of linear polarization as a function of phase angle (i.e. the angle between the directions to the Sun and to the observer, as seen from the target body).

The basic refraction and Fresnel laws imply that the plane of linear polarization of asteroids should always be perpendicular to the scattering plane (i.e. the plane containing the Sun, the object and the observer; see, for instance, Dollfus et al. 1989; Zellner \& Gradie 1976). However, the actual scattering mechanisms are more complicated than that. Coherent-backscattering (Muinonen et al. 2002) and single-particle scattering (Shkuratov et al. 1994, 2002; Lumme \& Rahola 1998) can produce polarization aligned with the scattering plane. As a consequence, the state of linear polarization of asteroids, as a rule, is found to be very close to a direction that is either perpendicular or parallel to the scattering plane, within the limits of accuracy of the measurements of the position angle of linear polarization. This property has led most authors to express the polarization state of asteroids and other atmosphereless Solar system bodies, in terms of the flux difference $P_{\mathrm{r}}$ between the scattered sunlight components having their polarization plane perpendicular and parallel to the scattering plane, normalized to their sum:

$$
P_{\mathrm{r}}=\frac{I_{\perp}-I_{\|}}{I_{\perp}+I_{\|}}
$$


According to this definition, $P_{\mathrm{r}}$ can be either positive or negative depending upon the (either parallel or perpendicular) orientation of the plane of linear polarization with respect to the scattering plane. The modulus of $P_{\mathrm{r}}$ is, in principle, coincident with the measured degree of linear polarization. In practical cases, it can be found to be slightly lower for reasons that might be the result of either measurement accuracy or the more complicated scattering properties of the surfaces that are beyond the scope of the present paper.

$P_{\mathrm{r}}$ has the advantage of conveying simultaneously information about the fraction of linear polarization and the orientation of the plane of polarization for atmosphereless Solar system objects when observed as point-like sources. The range of phase angle for which $P_{\mathrm{r}}$ is negative is commonly called the negative polarization branch.

In the past, progress in the field of asteroid polarimetry has been slowed down because of a general lack of dedicated instruments. In this paper, we describe the first results of a dedicated survey that makes use of a new polarimeter built at the Astrophysical Observatory of Torino, Italy. This work extends and improves some preliminary results presented in Devogèle et al. (2015).

\section{INSTRUMENT DESCRIPTION}

The determination of the degree of linear polarization requires the measurement of the intensities of light components produced by the splitting of an incident light beam. Therefore, an accurate measurement of linear polarization requires high signal-to-noise $(\mathrm{S} / \mathrm{N})$ photometric measurements, especially in cases, such as asteroid polarimetry, in which the fraction of linear polarization is intrinsically quite low (in most cases, below 1 per cent).

The splitting of the incoming light beam in linear polarization measurements is produced by optical devices such as Wollaston prisms. The polarimeter described in this paper is based on a wedged double Wollaston configuration (Oliva 1997). It has been designed by one of us (CP) and is described by Pernechele et al. (2012). This instrument is very similar to another designed by the same author, which in the past was operational at the Asiago Observatory, Italy (Fornasier et al. 2006), and is currently operational at the Nordic Optical Telescope (NOT) telescope at La Palma, Canary Islands, Spain (Fornasier, Belskaya \& Perna 2015). Our new Torino polarimeter (ToPol) instrument is installed at the Cassegrain focus (F/12.5) of the 1.04-m Omicron (West) telescope of the Centre Pédagogique Planète et Univers (C2PU) facility, located on the Calern plateau and managed by the Observatoire de la Côte d'Azur, France (IAU code, 010). The field of view is $5.31 \times 0.95 \mathrm{arcmin}^{2}$ for the ToPol instrument.

The new polarimeter is mainly devoted to carrying out a dedicated programme of asteroid observations: the Calern Asteroid Polarimetric Survey (CAPS).

Because of the use of a wedged double Wollaston as an analyser, both $Q / I$ and $U / I$ can be measured simultaneously with only one exposure. The wedged double Wollaston prism splits the incoming light beam into four separate components, corresponding to the $0^{\circ}, 90^{\circ}, 45^{\circ}$ and $135^{\circ}$ linear polarization states. It produces four simultaneous images on the focal plane, which are seen as clearly separated stripes on the CCD detector. An example of a ToPol frame is displayed in Fig. 1.

A schematic diagram and a photograph of the ToPol instrument are displayed in Fig. 2.

By measuring the photometric fluxes of the four polarized images, we obtain all the polarimetric information needed to compute $P_{\mathrm{r}}$, after some calibration steps (see Section 3).

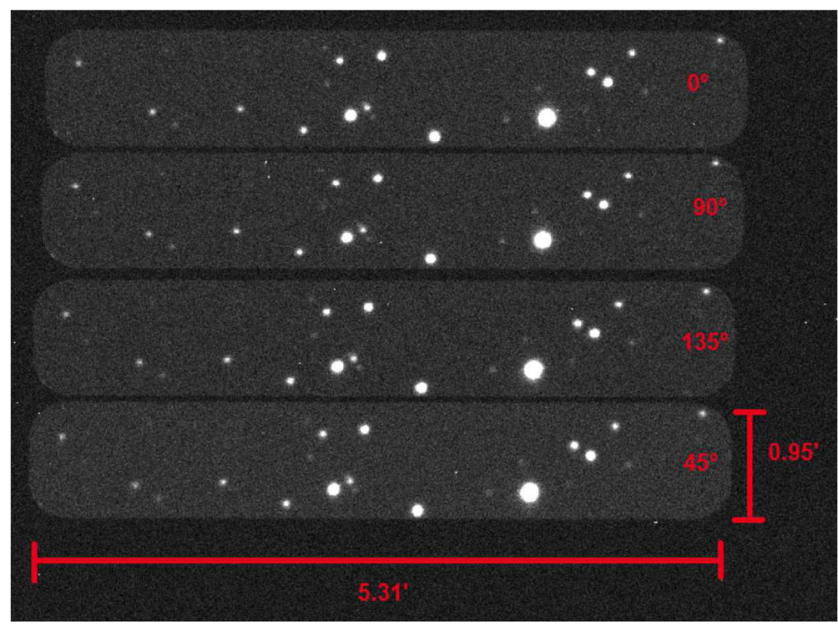

Figure 1. Example of a ToPol frame. The four strips correspond to the four images of the field of view of ToPol. Each strip corresponds to one specific polarization state: from top to bottom, $0^{\circ}, 90^{\circ}, 135^{\circ}$ and $45^{\circ}$, respectively.

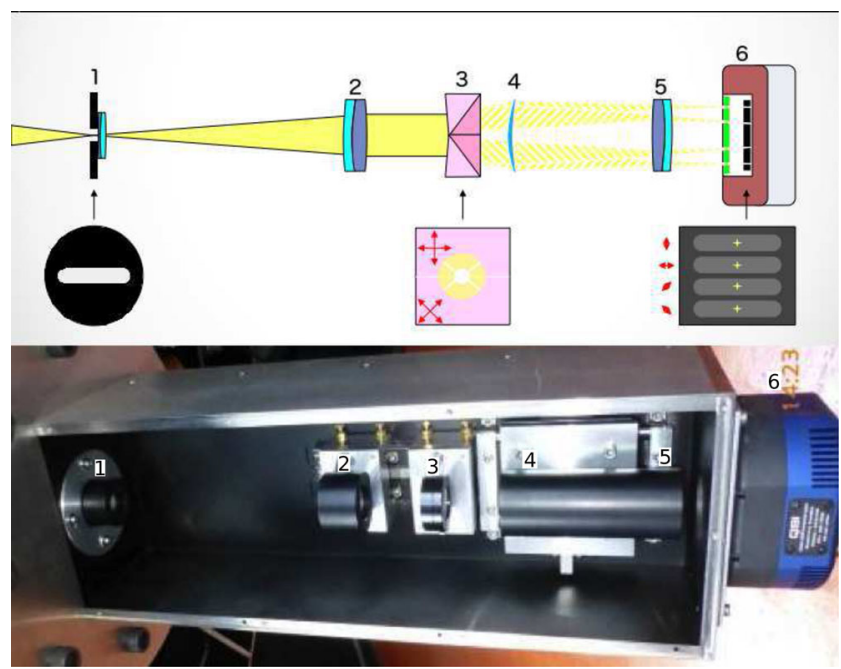

Figure 2. Schematic diagram and photograph of the ToPol instrument: 1, field stop followed by the field lens; 2, collimator lens; 3 , wedged double Wollaston; 4 and 5, camera optics; 6, QSI632 CCD camera.

The CCD camera attached to the ToPol instrument is a QSI632ws. It is equipped with a KAF-3200ME sensor of $2184 \times 1472$ pixels and a filter wheel with classical Johnson-Cousins UBVRI filters.

Analyses of flat-fields show that the CCD has currently no dead pixels. Dark frame analyses show that only 24 isolated pixels possess high dark current value.

\section{DATA REDUCTION PRINCIPLES FOR POLARIMETRY}

To measure the fraction of linear polarization of our targets, standard CCD reduction techniques are first applied (dark frame subtraction and flat-field correction). Then, aperture photometry is applied to all four separate subimages of the target. A growth curve strategy is applied to optimize the aperture sizes (see below). This yields the values of the four polarized components $I_{0}, I_{45}, I_{90}$ and $I_{135}$. The 
reduced Stokes parameters $q$ and $u$ are then computed according to their standard definitions (Shurcliff 1962):

$q=\frac{Q}{I}=\frac{I_{0}-I_{90}}{I_{0}+I_{90}}$

$u=\frac{U}{I}=\frac{I_{45}-I_{135}}{I_{45}+I_{135}}$.

Here, $I, Q$ and $U$ are the standard Stokes parameters. Finally, the total polarization degree $P$ and the position angle $\theta$ of the linear polarization plane, with respect to the instrument's zero direction, are obtained from

$P=\sqrt{q^{2}+u^{2}}$

and

$\theta=\frac{1}{2} \arctan \frac{u}{q}$.

Note that the relation between $P$ and the $P_{\mathrm{r}}$ parameter defined in Section 1 , which is usually adopted in polarimetric studies of small Solar system bodies, is simply

$P_{\mathrm{r}}=P \cos (2 \phi)$,

where $\phi$ is the angle between $\theta$ and the position angle of the plane perpendicular to the scattering plane (Zellner \& Gradie 1976).

The reduced Stokes parameters $q$ and $u$ are related to the instrument's internal reference plane, which is not necessarily aligned exactly along the standard reference direction for astronomical polarimetry (i.e. the celestial North direction according to IAU recommendations (IAU 1973)). Moreover, these parameters are likely to be biased by the instrumental response. Thus, a careful instrumental calibration is required (see Section 4).

\subsection{Curve of growth}

The fraction of linear polarization for main belt asteroids is relatively small (usually of the order of 1 percent or less, with the highest values of $P_{\mathrm{r}}$ rarely exceeding \pm 2 per cent). So, high photometric accuracy in the measurement of the four images of the target is required. Point spread function (PSF) fitting or optimal image subtraction techniques cannot be applied, as the field of view of the instrument is narrow and in general no suitable reference stars can be found in the field of view. Thus, aperture photometry is used to extract the four polarized fluxes. The measured values of the Stokes parameters are sensitive to the size of the photometric aperture. In order to obtain more accurate results, we adopt the 'curve of growth' approach suggested by Bagnulo et al. $(2011,2015)$. This technique consists of measuring the values $q$ and $u$ given by equations (2) and (3) as a function of the aperture. Usually, the $q$ and $u$ values converge to an asymptotic value much faster than the values of the flux on the individual apertures. The choice of the best aperture is a compromise between reaching a plateau and keeping the lowest possible error bar (which, at larger apertures, can be dominated by background noise, and hence increases with the aperture size). An example of such a curve of growth is given in Fig. 3 .

A first estimation of the sky-background is made by taking the average value of the pixels in an annulus around the target. The second step consists of building a curve of growth of the flux of the target (with sky-background subtracted). For great aperture radii, the curve of growth should become flat, as external pixels only contain background flux (with the assumption that the background

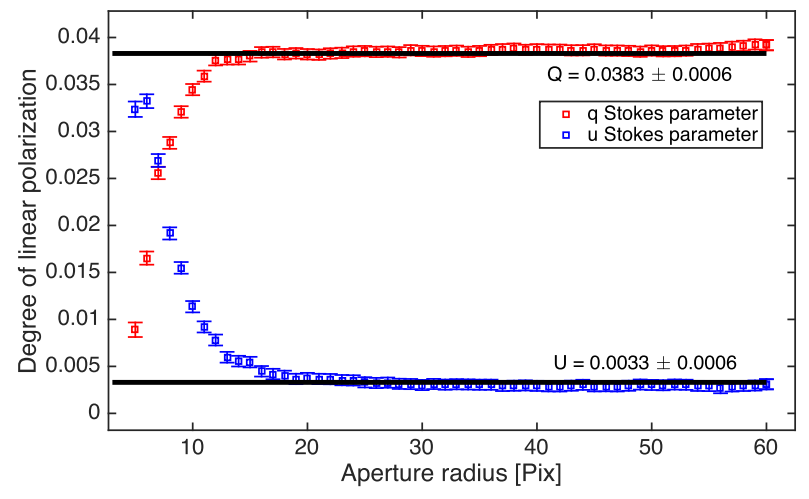

Figure 3. Example of a typical curve of growth. The red squares represent the $q$ reduced Stokes parameter as a function of the aperture radius (in pixels). The blue squares correspond to the $u$ reduced Stokes parameter. The black lines represent the best values of $q$ and $u$. The $u$ curve was reversed for display reasons.

is linear with pixel location). If this is not the case, then the skybackground is adjusted until the growth curve shows a flat trend. The correction to the sky-background is kept small and is smaller than three times the standard deviation of the sky-background pixel values.

\section{ASSESSMENT OF INSTRUMENTAL POLARIZATION AND ITS STABILITY}

The imperfections of the optical and mechanical properties of ToPol, as of any other polarimeter, produce spurious polarization of the incoming light. This spurious polarization, called instrumental polarization, is a result of the asymmetry on the optics and non-normal reflection effects, as well as artificial polarization effects resulting from different transmission coefficients along the light path of the four images of the source.

Because of the effect of instrumental polarization, the results of the polarimetric measurements are not simply the normalized Stokes parameters $q$ and $u$, but rather a couple of parameters $q^{\prime}$ and $u^{\prime}$ defined as

$q^{\prime} \simeq q+q_{0}$

and

$u^{\prime} \simeq u+u_{0}$

where $q_{0}$ and $u_{0}$ are a result of instrumental polarization effects.

Both $q_{0}$ and $u_{0}$ can be determined by means of measurements of standard unpolarized stars (for which the $q$ and $u$ parameters are supposed to be identically null), and they can then be subtracted from subsequent observations. In addition, the measurement of highly polarized, standard stars allows us to correct for the offset between the on-sky north direction and the zero instrumental direction. For a list of standard stars for linear polarization, see, for example, Hsu \& Breger (1982), Turnshek et al. (1990) and Gehrels (1974).

The stability over time of the instrumental polarization must be assessed by repeating the measurements of standard stars. In particular, the adopted observing procedures require that during each observing night at least two zero-polarization standard stars and one high-polarization standard star are measured (see Appendix A for extended tables of ToPol observations of standard stars).

However, the most important feature to be checked is not simply the absolute value of the instrumental bias affecting the determination of the $q$ and $u$ parameters, but the stability of this instrumental 
bias. The stability must be assessed over both short term (one single night) as well as over the long term (several nights in the same session, or even several sessions). Short-term stability of instrumental offsets is the minimal requirement for the calibration process to be reliable and accurate.

The stability of the instrumental polarization was determined by measuring 500 times the same standard polarized stars during an entire 8-h night (HD 90508, $m_{V}=6.5$; see Gehrels 1974). The standard deviation of these 500 measurements was found to be 1.1 $\times 10^{-4}$ for $q$ and $1.8 \times 10^{-4}$ for $u$. Thus, the instrumental stability for the short term (one night) does not appear to be a limiting factor for ToPol.

In 2015 February and March, eight full nights were devoted to measurements of instrumental polarization, and 17 unpolarized standard stars were observed. The observing circumstances of all the observations are given in Appendix A. From these measurements, we could determine with good accuracy the amount of instrumental polarization and its stability over a period of time of about one month. From these data, the instrumental polarization coefficients were found to be

$q_{0}^{(V)}=3.834 \times 10^{-2} \pm 1.0 \times 10^{-4}$

and

$u_{0}^{(V)}=3.04 \times 10^{-3} \pm 1.7 \times 10^{-4}$,

in the $V$ band, and

$q_{0}^{(R)}=3.641 \times 10^{-2} \pm 1.0 \times 10^{-4}$

and

$u_{0}^{(R)}=2.90 \times 10^{-3} \pm 1.9 \times 10^{-4}$,

in the $R$ band.

The measurements of nine highly polarized standard stars during the first year of observations showed that ToPol is rotated by 0.7 \pm 0.4 with respect to the IAU convention for the zero direction of polarization. The residuals between the known polarization for these standard stars and the polarization measured by ToPol after removal of the instrumental bias and rotation is $2 \times 10^{-4}$. However, these results have to be taken with caution. Indeed, it was reported by Hsu \& Breger (1982) and Bastien et al. $(1988,2007)$ that most of the stars that qualify as high-polarized standard stars may show variability in polarization.

\section{EXPECTED SIGNAL-TO-NOISE RATIO}

The expected errors on $q$ and $u$ can be computed using the following relation (Bagnulo et al. 2006):

$\sigma_{X}=\frac{1}{\sqrt{F}}$.

Here, $\sigma_{X}$ corresponds to the expected errors on the $q$ or $u$ parameter and $F$ is the total flux from the two beams used to compute $q$ or $u$. Based on equation (12), the expected error bars for observations of targets having different apparent magnitudes as a function of exposure time can be computed using an equation of the type

$\sigma_{X}=\frac{1}{\sqrt{t_{\mathrm{exp}} 10^{-0.4(m-a)}}}$,

where $t_{\exp }$ is the exposure time, $m$ is the magnitude of the target and $a$ is a parameter corresponding to the zero instrumental magnitude.

The $a$ parameter for the ToPol instrument attached to the Omicron 1.04-m telescope (ToPol @ C2PU) was empirically determined from

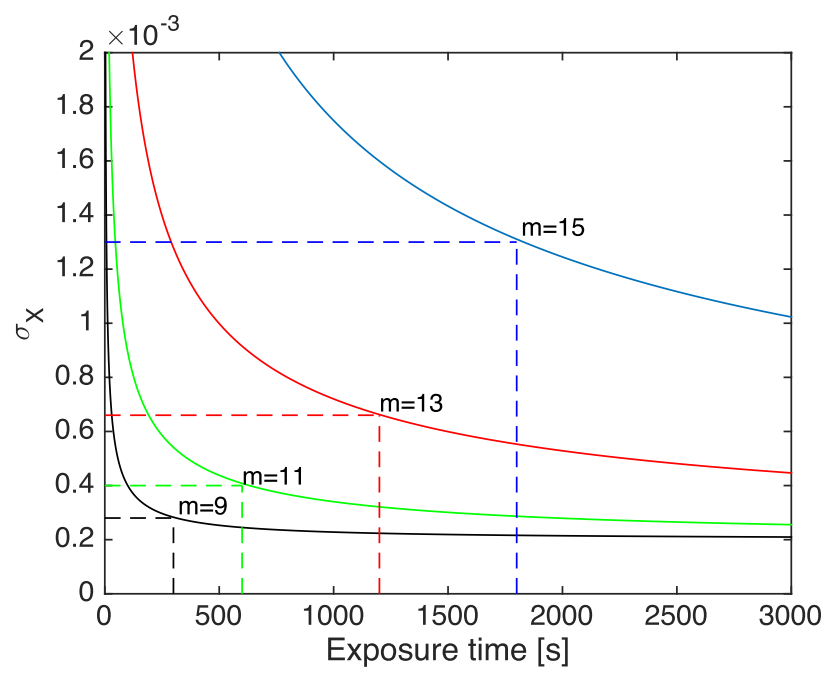

Figure 4. Expected error bar as a function of exposure time for the magnitudes of different targets. The dashed lines correspond to the typical cumulative exposure times used for different source magnitudes.

the observation of targets of different magnitude. A systematic error should be added to the result of equation (13) to take into account the error made on the estimation of the instrumental polarization. This error is estimated to be $2 \times 10^{-4}$. Fig. 4 shows the expected error bars as a function of exposure time and magnitude of the target.

Setting the limiting magnitude to 15, ToPol@C2PU can measure the full phase-polarization curve for more than 650 different asteroids. For a 15-mag asteroid, a 30-min cumulative exposure time will result in an accuracy of $1.3 \times 10^{-3}$. In the case of a limiting magnitude of 13 , the full phase-polarization of more than 160 asteroids can still be measured. The expected error bar on a 13-mag asteroid is $6.5 \times 10^{-4}$ with a cumulative exposure time of $20 \mathrm{~min}$. Finally, if we restrict ourselves to asteroids brighter than $11 \mathrm{mag}$, only a few more than 20 asteroids can be fully covered. However, the accuracy can be as good as $4 \times 10^{-4}$ with only 10-min exposure time. For brighter objects, the instrumental polarization become the dominant source of errors.

\section{FIRST RESULTS OF THE CALERN ASTEROID POLARIMETRIC SURVEY}

The preliminary data base of asteroid polarimetric measurements presented in this paper includes 124 entries, referring to a total of 78 asteroids observed in the first operational phase of CAPS. Most data consist of single measurements of objects in the standard Johnson $V$ filter, but several objects also have a measurement in standard $R$ colour, while in five cases only an $R$ measurement is available. The whole data base is given in Appendix B. For each measurement, we list the asteroid's number, the observation date, the corresponding phase angle, $P_{\mathrm{r}}$ and its associated uncertainty (both in units of per cent) and the adopted filter, respectively. Generally, we have so far obtained only one or two different polarization measurements per object.

For some asteroids of our sample, the linear polarization had never been measured before, or very rarely. Some objects have a reliable estimate of the geometric albedo, and they can be used to calibrate the existing relations between albedo and polarimetric behaviour; see Cellino et al. (2015b) for further details. Some objects 

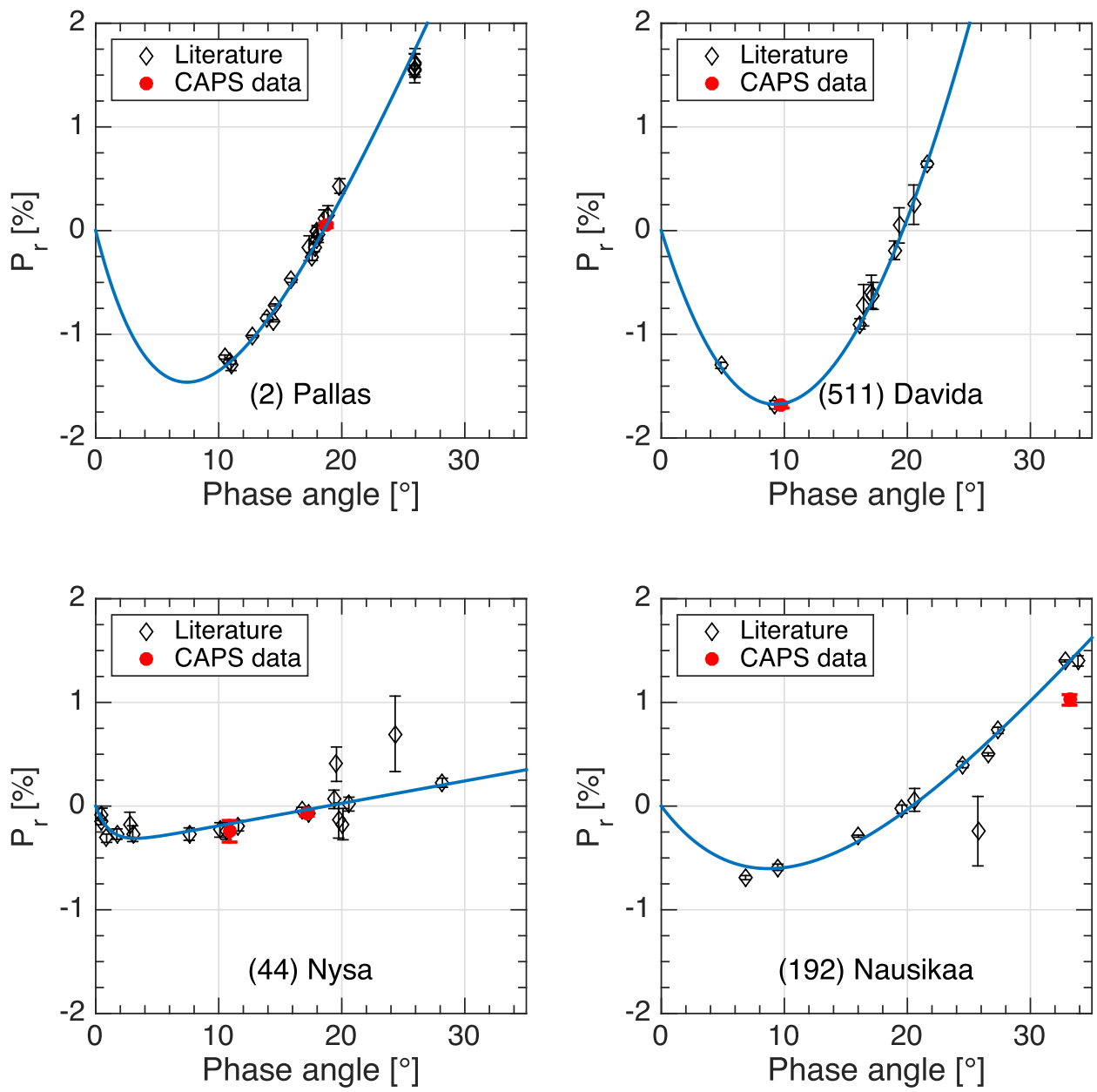

Figure 5. Phase-polarization curves for asteroids (2) Pallas, (44) Nysa, (192) Nausikaa and (511) Davida. Open symbols denote data available in the literature and full symbols represent new CAPS data. The best-fitting curves have been computed according to equation (14).

might also belong to dynamical families. Our reference for this, among differently published family lists, is Milani et al. (2014).

In what follows, we limit our analysis to polarimetric measurements obtained in $V$. When browsing the published $V$ polarimetric data available in the literature, in order to compare them with our CAPS data, we selected only measurements having uncertainties no larger than 0.2 per cent, as CAPS data are generally of a much better quality. As for the literature data, we used the polarimetric data bases available at the Planetary Data System (PDS) web site. ${ }^{1}$ Moreover, we also used some polarimetric measurements obtained at the CASLEO observatory, Argentina, and published by Gil Hutton, Cellino \& Bendjoya (2014).

Asteroid phase-polarization curves generally have a common morphology, characterized by a negative polarization branch followed, after an inversion angle $\alpha_{\text {inv }}$ (generally at a phase angle around $20^{\circ}$ ), by a linear increase of $P_{\mathrm{r}}$. They can be suitably represented by the following relation (Muinonen et al. 2009):

$P_{\mathrm{r}}(\alpha)=A\left[\mathrm{e}^{(-\alpha / B)}-1\right]+C \alpha$.

Here, $\alpha$ is the phase angle and $A, B$ and $C$ are parameters to be derived by least-squares techniques (see Cellino et al. 2015b, 2016).

\footnotetext{
${ }^{1}$ The data are available at http://pds.jpl.nasa.gov (files maintained by D. F. Lupishko and I. N. Belskaya).
}

We used the above relation to compute best fits of the available data for objects for which a sufficient number of measurements are available, as shown in some of the figures presented below.

CAPS data can be classified in different categories. First, there are previously well-observed (and generally bright) asteroids for which the new CAPS data are found to be in excellent agreement with previously derived phase-polarization curves. There is only one exception of a single CAPS measurement of asteroid (192) Nausikaa, which is found to show a lower value of positive polarization at a phase angle of about $33^{\circ}$, with respect to previously available measurements. The nominal error bars of CAPS data are comparable with, and often better than, most data available in the literature. Four cases, including the discrepant case of Nausikaa, are shown in Fig. 5.

Our data base includes a large variety of asteroids belonging to different taxonomic classes. As is well known, two major taxonomic complexes, $S$ and $C$, dominate the asteroid main-belt population, and can also be split into a number of separate subclasses (see, for instance, Bus \& Binzel 2002). S-class asteroids have moderate albedo and orbit mostly in the inner region of the belt, so it is not unexpected that they account for a large fraction of the objects observed so far by CAPS. In particular, if we consider only asteroids for which measurements in standard $V$ are available, 25 of these (out of 74) belong unambiguously to the $S$-complex. Our polarimetric measurements for asteroids (26), (57), (61) and (103) are the first 


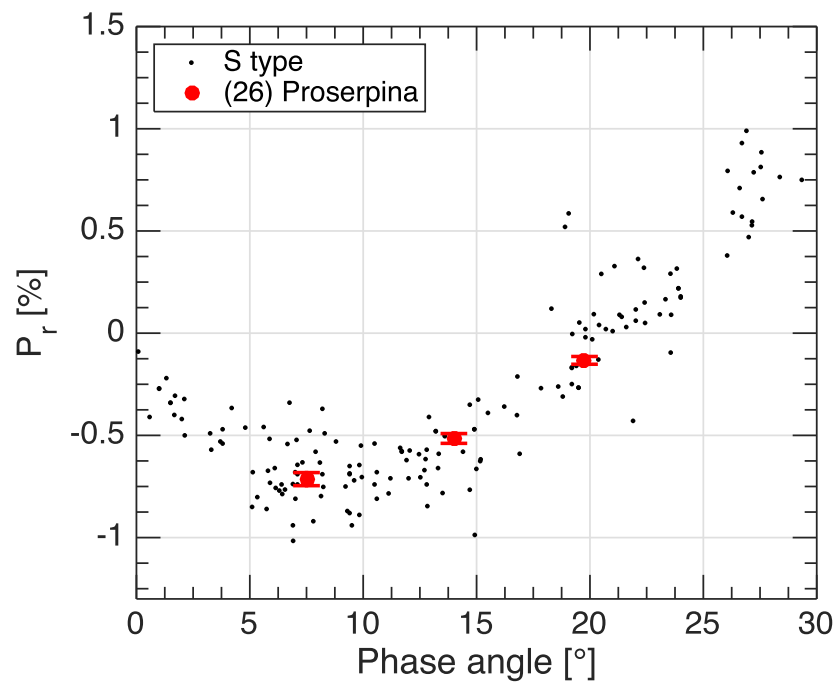

Figure 6. CAPS phase-polarization data for the $S$-class asteroid (26) Proserpina (three larger points shown in red), to be compared with all data previously available in the literature for the other asteroids belonging to this taxonomic class (small black points). No previous polarimetric data had been obtained for this asteroid

data obtained for these objects, and share the known polarimetric behaviour already known for objects of this class, as shown, as an example, in Fig. 6 in the case of the asteroid (26) Proserpina.

Some other $S$-class asteroids had several polarimetric measurements already available in the literature, and the new CAPS data are consistent with these. In some cases, the new data improve the phase coverage, allowing us to derive best-fitting curves according to equation (14).

We also obtained one single measurement for the asteroid (71) Niobe, whose taxonomic classification is uncertain, the old $S$-class proposed by Tholen (1984) having been more recently unconfirmed by Bus \& Binzel (2002), who instead propose an Xe classification (see also Fornasier et al. 2006). The CAPS single measurement obtained at a phase angle of 7.6 is not decisive, being marginally compatible with both classifications.

Asteroids belonging to the $C$-class have low albedo and dominate the asteroid inventory in the outer regions of the main belt. $C$-class asteroids are intrinsically fainter than $S$-class objects of the same size. Nevertheless, these objects are as abundant as $S$-class asteroids in the CAPS data base (in $V$ colour), including 24 objects out of 74 . Also in this case, CAPS data fit nicely and extend the data available in the literature for the objects already observed in the past. As an example, we show in Fig. 7 the resulting phase-polarization curve of asteroid (51) Nemausa

Many other taxonomic classes are already included in the CAPS data base, and are an excellent base for a future, more systematic study of the polarimetric properties of objects belonging to different taxonomic classes.

The obtained results make us confident that CAPS measurements are of a very good quality (note that in Fig. 5 the nominal error bars of the CAPS data are generally smaller than the size of the symbols used in the plot), and they encourage us to use them to analyse the behaviour of asteroids for which previously available polarimetric data were scarce and/or of poor quality.

Many objects in the CAPS data base were poorly observed in the past. For some of them, our new data fill gaps in the existing phase-polarization curves, making it possible to improve them significantly or even to derive for the first time a reasonable fit using

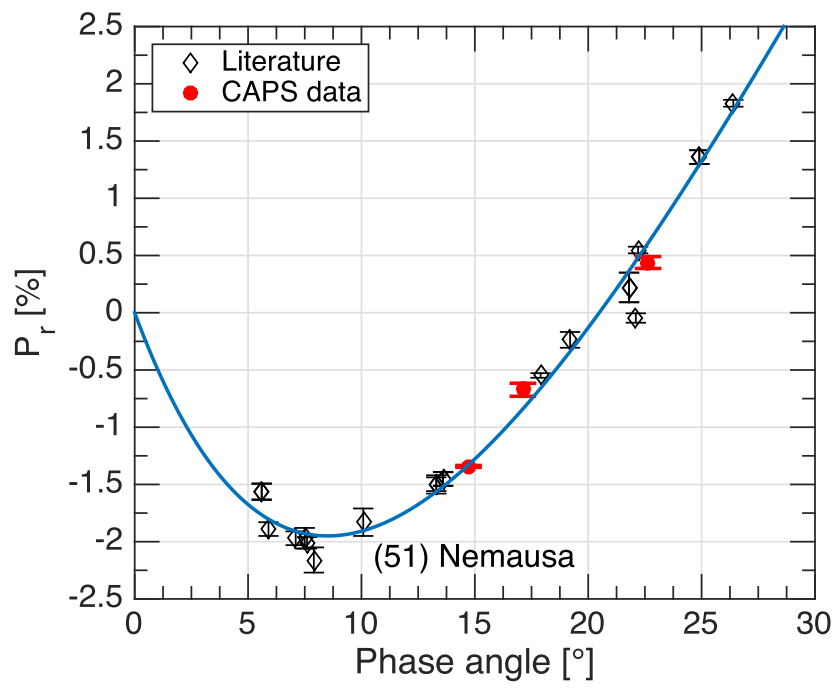

Figure 7. Phase-polarization curve of the asteroid (51) Nemausa: open symbols, data available in the literature; solid red symbols, new CAPS data. The best-fitting curve has been computed according to equation (14).

equation (14). A couple of cases are shown as an example in Fig. 8. For other objects, there are only a very few published measurements. For them, CAPS data are an important addition, but still not sufficient to compute a best-fitting phase-polarization curve using equation (14). The case of (532) Herculina is shown, as an example, in Fig. 9. Finally, for some objects including asteroids (26), (35), (38), (45), (57), (61), (96), (191), (194), (273), (304), (313), (426), (500), (783), (824), (1372) and (1702) - the latter being affected by a large measurement uncertainty - our CAPS data are the very first existing polarimetric measurements.

In what follows, we make a short case-by-case description of some interesting results obtained for a small number of objects of particular interest. Some of them are also shown in Figs 8, 9 and 10.

\section{1 (45) Eugenia}

(45) Eugenia was one of the first asteroids found to be a binary system using the Adaptive Optics observations of Merline et al. (1999). It was classified as an $F C$-class asteroid according to old classifications (Gradie \& Tedesco 1982), whereas it is classified as $C$ according to the Small Main-Belt Asteroid Spectroscopic Survey (SMASS; Bus \& Binzel 2002). We have two measurements of Eugenia at a phase angle of about $20^{\circ}$, shown in Fig. 8. Both measurements indicate that, at this phase angle, (45) Eugenia is already deeply in the positive polarization branch, suggesting a low value of the inversion angle. This is exactly the polarimetric property characterizing the $F$ class, according to Belskaya et al. (2005). According to CAPS results, therefore, the most correct taxonomic classification of (45) Eugenia should be $F$, a class that has been lost in the most recent spectroscopic surveys, based on data no longer covering the blue part of the reflectance spectrum (see also Cellino et al. 2015a) We also note that the small companion of (45) Eugenia is much fainter, and its contribution to the measured flux should be negligible.

\section{2 (96) Aegle}

This asteroid is the lowest-numbered member of a dynamical family found by Milani et al. (2014). It is also classified as belonging to 

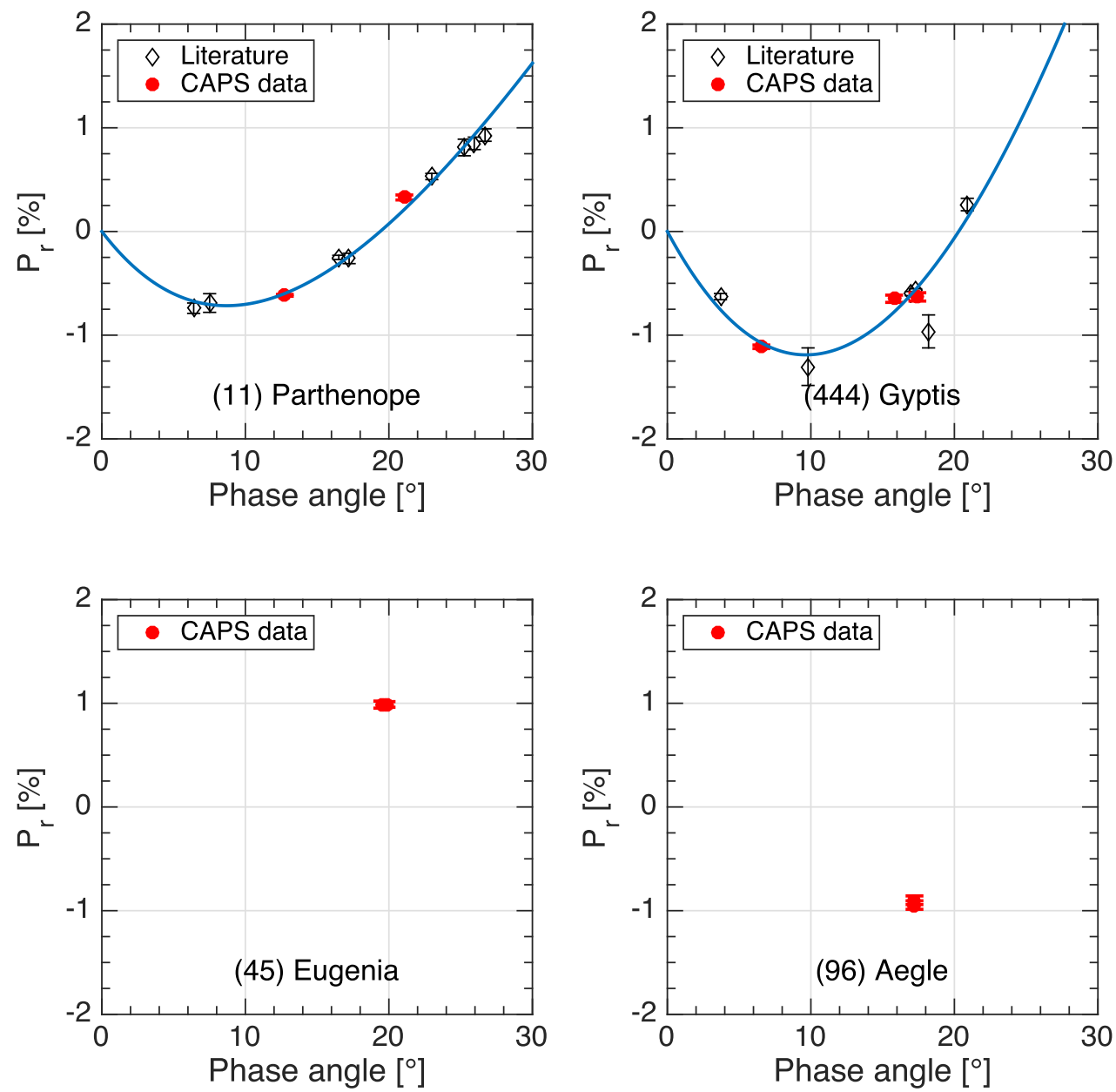

Figure 8. Phase-polarization curves for asteroids (11) Parthenope and (444) Gyptis, and new phase-polarization data for (45) Eugenia and (96) Aegle: open symbols, data available in the literature; solid symbols, new CAPS data.

the rare $T$ taxonomic class. As far as we know, our two CAPS measurements, shown in Fig. 8, are the only polarimetric data currently available for asteroids of this taxonomic class. The obtained values, obtained at a phase angle of $17^{\circ}$, are diagnostic of a low albedo.

\section{3 (194) Prokne}

Prokne is another case of the lowest-numbered member of a dynamical family identified by Milani et al. (2014). We have so far two CAPS measurements in $V$ light for Prokne, one at a phase angle close to $12^{\circ}$ and the other around $25^{\circ}$. (shown in Fig. 9). These two measurements, though not yet sufficient to produce a fit of the phase-polarization curve by means of equation (14), clearly indicate that this should be a low-albedo asteroid, being characterized by a deep negative polarization branch (compare also with polarimetric data for the low-albedo $C$-class asteroids shown as a background in Fig. 10). Thus, we confirm the $C$ taxonomic classification (Tholen 1984) and the low-albedo value found for this object from Wide-field Infrared Survey Explorer (WISE) thermal radiometry data (Masiero et al. 2011). Note that a low-albedo value for Prokne strongly suggests that this asteroid should be considered as a likely interloper in its own family, because the average albedo value of the family is higher, of the order of 0.15 according to WISE data.

\section{4 (444) Gyptis}

This $C$-class asteroid has nothing special per se. However, because we have an excellent knowledge of its size, derived by stellar occultations, Gyptis is included in the list of asteroids with reliable values of the geometric albedo published by Shevchenko \& Tedesco (2006). Several objects in this list have been recently analysed by Cellino et al. (2015b) to derive more accurate values of the calibration coefficients appearing in differently proposed relations between geometric albedo and polarimetric properties. The new CAPS measurements, shown in Fig. 8, greatly improve the previously poor phase-polarization curve of this asteroid.

\section{5 (500) Selinur}

No taxonomic classification has been proposed so far in the literature for this asteroid. Our single value of $P_{\mathrm{r}} \simeq-0.6$ per cent at a phase value close to $8^{\circ}$, shown in Fig. 9, suggests that Selinur should have a fairly high albedo.

\section{6 (1372) Haremari}

(1372) Haremari is a fairly small asteroid, about $20-30 \mathrm{~km}$ in size according to different estimations based on thermal radiometry measurements by the IRAS and WISE satellites (Tedesco et al. 2002; 

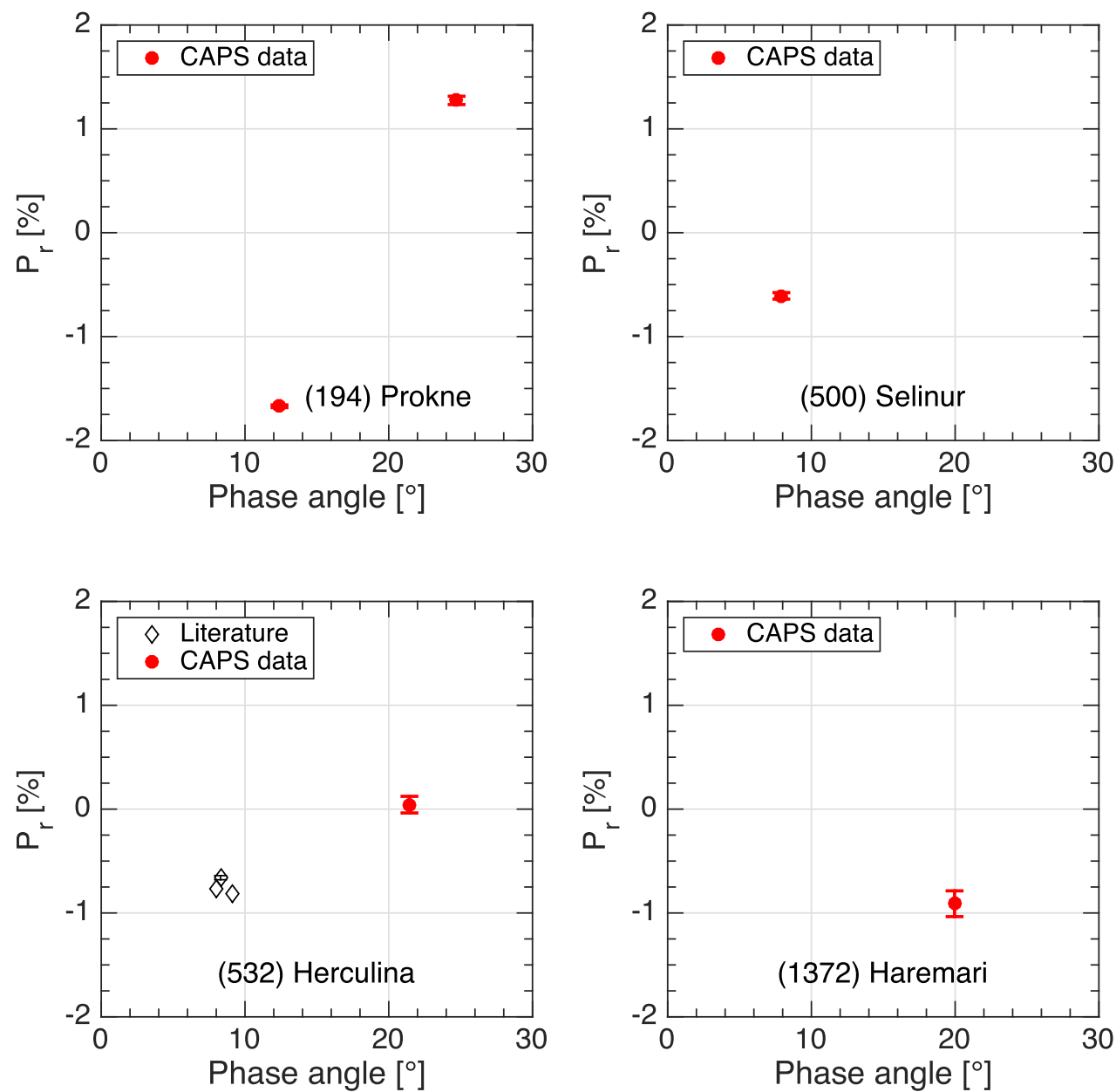

Figure 9. Phase-polarization data for asteroids (194) Prokne, (500) Selinur, (532) Herculina and (1342) Haremari: open diamonds, data available in the literature for (532) Herculina; full symbols, new CAPS data.

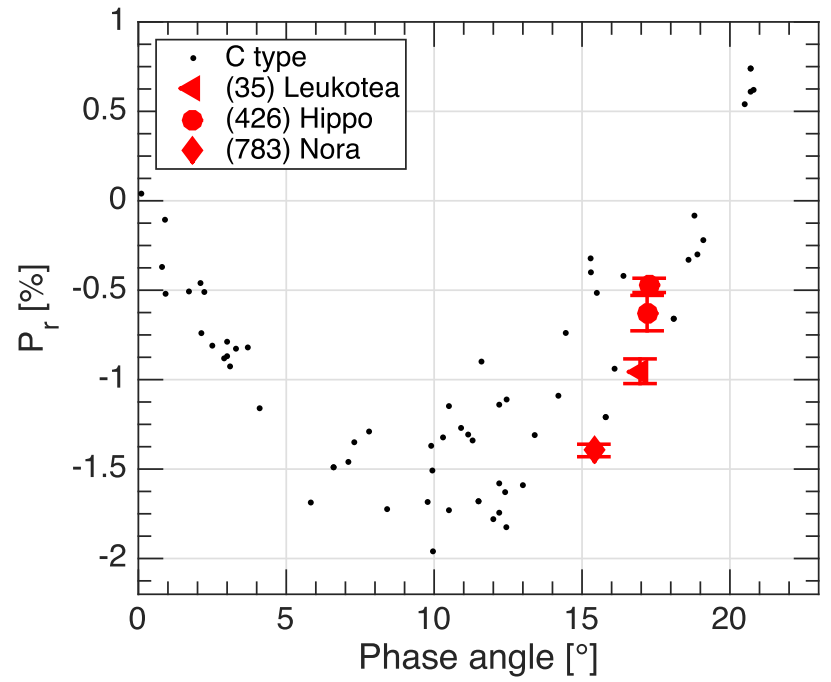

Figure 10. CAPS phase-polarization data for asteroids (35) Leukotea, (426) Hippo and (783) Nora (large symbols), to be compared with all data available in the literature for asteroids belonging to the $C$ taxonomic class (small points).
Masiero et al. 2011). It belongs to the dynamical family of Watsonia (Novaković, Cellino \& Knežević 2011; Milani et al. 2014). This is the first family that has been found by Cellino et al. (2014) to consist of Barbarian asteroids. These objects exhibit anomalous phase-polarization curves, characterized by a very wide extension of the negative polarization branch up to values near $30^{\circ}$. Several Barbarians also show reflectance spectra, suggesting very high abundances of the spinel mineral on their surface (Sunshine et al. 2008). Spinel is a refractory compound found in the socalled calcium-aluminium-rich inclusions (CAIs) in chondritic meteorites. Therefore, Barbarian asteroids might be the remnants of the very first generation of planetesimals accreted in the Solar system. Our single polarimetric measurement of Haremari, shown in Fig. 9, shows $P_{\mathrm{r}}=-0.911 \pm 0.124$ percent at a phase angle of 19.96, which confirms that this asteroid must now be added to the still small list of known Barbarians.

As for other Barbarians, we note that so far we have also obtained a few new polarimetric measurements for asteroids (234) Barbara (the prototype of this class), (236) Honoria and (679) Pax. We plan to devote as much time as possible in the future to observations of these asteroids. We will present current and future CAPS data for Barbarians in a separate, dedicated paper. 


\subsection{A few slow rotators}

Asteroids (35) Leukothea, (426) Hippo and (783) Nora are three slow rotators, with periods of $31.900,34.3$ and $34.4 \mathrm{~h}$, respectively. Leukothea is classified as $C$ by different authors. For Hippo, we have at disposal only an old Tholen classification as $F$, whereas the classification of Nora is $C$ according to Bus \& Binzel (2002). As mentioned above, the $F$ class, first defined by Gradie $\&$ Tedesco (1982), is characterized, in polarimetric studies, by quite low values of the inversion angle, and a correspondingly narrow extension of the negative polarization branch. So far, we have obtained one polarimetric measurement for both Leukothea and Nora, and two observations for Hippo. All these measurements are in the negative polarization branch, and are shown in Fig. 10. In the case of (35) Leukothea and (783) Nora, we find $P_{\mathrm{r}}$ values that are more negative (though not dramatically) with respect to the usual values for most $C$-class asteroids observed at the same phase angles, as shown in Fig. 10. In the case of (426) Hippo, the two values of $P_{\mathrm{r}}=-0.628$ \pm 0.099 per cent and $P_{\mathrm{r}}=-0.473 \pm 0.040$ per cent at phase angles of 17.20 and 17.27 , respectively, are also more negative than one would expect for an $F$-class asteroid, which should normally exhibit

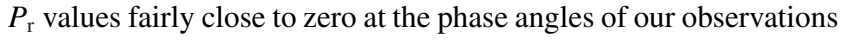
of Hippo. However, these two measurements also seem to suggest a fast 'race' to the inversion angle. In all three cases, the measured values of $P_{\mathrm{r}}$ are, in some respects, slightly more negative than we would expect. We note that all the above three objects are slow rotators. This might be just a coincidence, but we know that several Barbarian asteroids, which exhibit values of $P_{\mathrm{r}}$ more negative than normal in a wide range of phase angles, are also known to be slow rotators, as in the cases of (234) Barbara and (1372) Haremari, whose spin periods are longer than $15 \mathrm{~h}$. It is difficult to imagine a possible direct relation between slow rotation and unusual polarization properties. However, we cannot rule out the possibility that both the rotation state and surface properties might be, in some way, a consequence of age. The hypothesis of a relation between slow rotation and polarimetric properties is something that can be explored by carrying out a dedicated telescope survey such as CAPS.

\section{CONCLUSIONS}

We can summarize briefly the results obtained in the present analysis.

(i) The ToPol is characterized by a comparatively high, but stable instrumental polarization.

(ii) The results obtained so far for asteroids are in agreement with those previously published in the literature. In the case of poorly observed asteroids, CAPS measurements can already be used to improve significantly the coverage in phase angle. Many of these objects can be used to calibrate the relations between geometric albedo and polarimetric properties.

(iii) In a number of cases, the very preliminary results obtained so far are important as they concern objects that are particularly interesting because of rare taxonomic classifications, family membership, unusual rotation rates and/or peculiar polarimetric properties (Barbarian asteroids).

(iv) By carrying out our CAPS, we expect to be able to improve significantly the data set of asteroid polarization data, and to explore systematically some interesting branches of research, including Barbarians and a possible relation between slow rotation and unusual polarization properties, to be possibly interpreted in terms of evolutionary processes.
Based on the preliminary results presented in this paper, we are convinced that the CAPS can play an important role for asteroid studies in the years to come. Moreover, we also plan to use the new polarimeter to perform polarimetric studies of other Solar system bodies, including major planets and the Moon. Some preliminary results we have already obtained will be published in separate papers.

\section{ACKNOWLEDGEMENTS}

The ToPol was built at the INAF - Torino Astrophysical Observatory and funded by INAF in the framework of INAF PRIN 2009. Part of this work by MD was supported by the COST Action MP1104 'Polarization as a tool to study the Solar system and beyond'. The authors express their gratitude to the technical and administrative staff of the Calern observing station for their help and kind support.

\section{REFERENCES}

Bagnulo S., Boehnhardt H., Muinonen K., Kolokolova L., Belskaya I., Barucci M. A., 2006, A\&A, 450, 1239

Bagnulo S., Belskaya I., Boehnhardt H., Kolokolova L., Muinonen K., Sterzik M., Tozzi G.-P., 2011, J. Quant. Spectros. Radiat. Trans., 112, 2059

Bagnulo S., Cellino A., Sterzik M. F., 2015, MNRAS, 446, L11

Bailey J., Hough J., 1982, PASP, 94, 618

Bastien P., Drissen L., Menard F., Moffat A. F. J., Robert C., St-Louis N., 1988, AJ, 95, 900

Bastien P., Vernet E., Drissen L., Menard F., Moffat A. F. J., Robert C., St-Louis N., 2007, ASP Conf. Series Vol. 364, The Future of Photometric, Spectrophotometric and Polarimetric Standardization. Astron. Soc. Pac., San Francisco, p. 529

Belskaya I. N. et al., 2005, Icarus, 178, 213

Belskaya I. N., Cellino A., Gil-Hutton R., Muinonen K., Shkuratov Y., 2015, in Michel P., DeMeo F., Bottke W. F., eds, Asteroids IV. Univ. of Arizona Press, Tucson, p. 151

Bus S., Binzel R. P., 2002, Icarus, 158, 146

Cellino A., Belskaya I. N., Bendjoya Ph., di Martino M., Gil-Hutton R., Muinonen K., Tedesco E. F., 2006, Icarus, 180, 565

Cellino A., Bagnulo S., Tanga P., Novaković B., Delbò M., 2014, MNRAS, 439, L75

Cellino A., Gil-Hutton R., Belskaya I. N., 2015a, in Kolokolova L., Hough J., Levasseur-Regourd A.-C., eds, Polarimetry of Stars and Planetary Systems. Cambridge Univ. Press, Cambridge, p. 360

Cellino A., Bagnulo S., Gil-Hutton R., Tanga P., Cañada-assandri M., Tedesco E. F., 2015b, MNRAS, 451, 3473

Cellino A., Bagnulo S., Gil-Hutton R., Tanga P., Cañada-assandri M., Tedesco E. F., 2016, MNRAS, 455, 2091

Clemens D., Tapia S., 1990, PASP, 102, 179

Devogèle M. et al., 2015, European Planetary Science Congress 2015, EPSC2015-469

Dollfus A., Wolff M., Geake J. E., Lupishko D. F., Dougherty L., 1989, in Binzel R. P., Gehrels T., Matthews M. S., eds, Asteroids II. Univ. of Arizona Press, Tucson, p. 594

Fornasier S., Belskaya I. N., Shkuratov Yu. G., Pernechele C., Barbieri C., Giro E., Navasardyan H., 2006, A\&A, 455, 371

Fornasier S., Belskaya I. N., Perna D., 2015, Icarus, 250, 280

Gehrels T., 1974, Planets, Stars and Nebulae Studied with Photopolarimetry. University of Arizona Press, Tuscon

Gil Hutton R., Cellino A., Bendjoya Ph., 2014, A\&A, 569, A122

Gradie J., Tedesco E. F., 1982, Science, 216, 1405

Hiltner W. A., 1951, AJ, 114, 241

Hsu J.-C., Breger M., 1982, ApJ, 262, 732

International Astronomical Union, 1973, Transactions of the IAU, 15B, 166

Lumme K., Rahola J., 1998, J. Quant. Spectros. Radiat. Trans., 60439 
Masiero J. et al., 2011, ApJ, 741, 68

Merline W. et al., 1999, Nature, 401, 565

Milani A., Cellino A., Knežević Z., Novaković B., Spoto F., Paolicchi P., 2014, Icarus, 239, 46

Muinonen K., Piironen J., Shkuratov Y. G., Ovcharenko A. A., Clark B. E., 2002, in Bottke W. F. Jr, Cellino A., Paolicchi P., Binzel R. P., eds, Asteroids III. University of Arizona Press, Tucson, p. 123

Muinonen K., Penttilä A., Cellino A., Belskaya I. N., Delbò M., LevasseurRegourd A. C., Tedesco E. F., 2009, Meteoritics and Planet. Sci., 44 1937

Novaković B., Cellino A., Knežević Z., 2011, Icarus, 216, 69

Oliva E., 1997, A\&ASS, 123, 589

Pernechele C., Abe L., Bendjoya Ph., Cellino A., Massone G., Tanga P., 2012, Proc. SPIE, 8446, 84462

Petrova E. V., Tishkovets V. P., Jockers K., 2004, Solar System Research, 38,354

Schmidt G., Elston R., Lupie O. L., 1992, AJ, 104, 1563

Schulz A., Lenzen R., 1983, A\&A, 121, 158
Shevchenko V. G., Tedesco E. F., 2006, Icarus, 184, 211

Shkuratov Yu. G. et al., 1994, Earth, Moon, and Planets, 65, 201

Shkuratov Yu. G. et al., 2002, Icarus, 159, 396

Shurcliff W. A., 1962, Polarized Light. Harvard University Press, Cambridge, MA

Sunshine J., Connolly H. C., Mc Coy, Bus S. J., La Croix L. M., 2008, Science, 320, 514

Tedesco E. F., Noah P. V., Noah M., Price S. D., 2002, AJ, 123, 1056

Tholen D. J., 1984, PhD Thesis, University of Arizona

Turnshek D. A., Bohlin R. C., Williamson R. L. II, Lupie O. L., Koornneef J., Morgan D. H., 1990, AJ, 99, 1243

Wolff M., Nordsieck K., Nook M., 1996, AJ, 111, 856

Zellner B., Gradie J., 1976, AJ, 81, 262

\section{APPENDIX A: SUMMARY OF THE OBSERVATIONS OF STANDARD CALIBRATION STARS}

Table A1. Summary table of all observations for unpolarized standard stars. The first column gives the name of the star. The third column reports the magnitude in the respective filter (second column). $N_{\text {obs }}$ represents the number of observed frames, $t_{\text {exp }}$ is the exposure time of the individual frames, $q_{0}$ is the measured instrumental $q$ reduced Stokes parameter and $u_{0}$ is the measured instrumental $u$ reduced Stokes parameter.

\begin{tabular}{|c|c|c|c|c|c|c|c|}
\hline Star & Filter & Mag & Obs date & $N_{\text {obs }}$ & $\begin{array}{r}t_{\exp } \\
(\mathrm{s})\end{array}$ & $\begin{array}{c}q_{0} \\
\left(\times 10^{-2}\right)\end{array}$ & $\begin{array}{c}u_{0} \\
\left(\times 10^{-2}\right)\end{array}$ \\
\hline HD 10476 & $V$ & 5.24 & 2015/10/14 & 91 & 0.5 & $3.801 \pm 0.075$ & $-0.472 \pm 0.008$ \\
\hline HD 10476 & $V$ & 5.24 & $2015 / 10 / 15$ & 30 & 1 & $3.773 \pm 0.073$ & $-0.454 \pm 0.009$ \\
\hline HD 10476 & $V$ & 5.24 & $2015 / 12 / 08$ & 34 & 0.2 & $3.756 \pm 0.012$ & $-0.664 \pm 0.008$ \\
\hline HD 10476 & $V$ & 5.24 & $2015 / 12 / 09$ & 30 & 0.1 & $3.778 \pm 0.016$ & $-0.534 \pm 0.015$ \\
\hline HD 10476 & $V$ & 5.24 & $2015 / 12 / 10$ & 30 & 0.1 & $3.734 \pm 0.022$ & $-0.733 \pm 0.012$ \\
\hline HD 10476 & $V$ & 5.24 & $2015 / 12 / 11$ & 30 & 0.5 & $3.742 \pm 0.053$ & $-0.644 \pm 0.013$ \\
\hline HD 18803 & $V$ & 6.62 & $2015 / 02 / 19$ & 20 & 0.5 & $3.834 \pm 0.018$ & $-0.371 \pm 0.037$ \\
\hline HD 18803 & $R$ & 6.2 & $2015 / 02 / 19$ & 24 & 0.5 & $3.642 \pm 0.015$ & $-0.272 \pm 0.016$ \\
\hline HD 18803 & $V$ & 6.62 & $2015 / 10 / 15$ & 20 & 2 & $3.754 \pm 0.083$ & $-0.443 \pm 0.008$ \\
\hline HD 18803 & $V$ & 6.62 & $2015 / 12 / 10$ & 31 & 0.5 & $3.723 \pm 0.023$ & $-0.529 \pm 0.018$ \\
\hline HD 20630 & $V$ & 4.85 & $2015 / 02 / 19$ & 41 & 0.1 & $3.872 \pm 0.019$ & $-0.458 \pm 0.032$ \\
\hline HD 20630 & $R$ & 4.27 & $2015 / 02 / 19$ & 20 & 0.1 & $3.654 \pm 0.014$ & $-0.332 \pm 0.021$ \\
\hline HD 20630 & $V$ & 4.85 & $2015 / 02 / 22$ & 23 & 1 & $3.792 \pm 0.012$ & $-0.258 \pm 0.008$ \\
\hline HD 20630 & $R$ & 4.27 & $2015 / 02 / 22$ & 21 & 1 & $3.613 \pm 0.013$ & $-0.234 \pm 0.063$ \\
\hline HD 39587 & $V$ & 4.40 & $2015 / 02 / 22$ & 46 & 0.2 & $3.897 \pm 0.013$ & $-0.239 \pm 0.014$ \\
\hline HD 39587 & $R$ & 3.9 & $2015 / 02 / 22$ & 24 & 0.2 & $3.667 \pm 0.015$ & $-0.290 \pm 0.080$ \\
\hline HD 39587 & $V$ & 4.40 & $2015 / 10 / 16$ & 30 & 0.05 & $3.881 \pm 0.068$ & $-0.182 \pm 0.008$ \\
\hline HD 39587 & $V$ & 4.40 & $2015 / 12 / 11$ & 30 & 0.2 & $3.813 \pm 0.013$ & $-0.532 \pm 0.018$ \\
\hline HD 42807 & $V$ & 6.44 & $2015 / 02 / 18$ & 22 & 0.5 & $3.751 \pm 0.014$ & $-0.261 \pm 0.018$ \\
\hline HD 42807 & $R$ & 6.0 & $2015 / 02 / 18$ & 21 & 0.5 & $3.584 \pm 0.014$ & $-0.300 \pm 0.017$ \\
\hline HD 42807 & $V$ & 6.44 & $2015 / 02 / 22$ & 21 & 0.5 & $3.836 \pm 0.018$ & $-0.311 \pm 0.032$ \\
\hline HD 42807 & $R$ & 6.0 & $2015 / 02 / 22$ & 20 & 0.5 & $3.700 \pm 0.014$ & $-0.258 \pm 0.019$ \\
\hline HD 42807 & $V$ & 6.44 & $2015 / 12 / 09$ & 30 & 1 & $3.731 \pm 0.014$ & $-0.343 \pm 0.011$ \\
\hline HD 65583 & $V$ & 7.00 & $2015 / 02 / 18$ & 20 & 1 & $3.810 \pm 0.028$ & $-0.334 \pm 0.021$ \\
\hline HD 65583 & $R$ & 6.4 & $2015 / 02 / 18$ & 20 & 1 & $3.661 \pm 0.018$ & $-0.301 \pm 0.021$ \\
\hline HD 65583 & $V$ & 7.00 & $2015 / 02 / 22$ & 20 & 0.5 & $3.826 \pm 0.018$ & $-0.216 \pm 0.026$ \\
\hline HD 65583 & $R$ & 6.4 & $2015 / 02 / 22$ & 23 & 0.5 & $3.631 \pm 0.024$ & $-0.242 \pm 0.023$ \\
\hline HD 65583 & $V$ & 7.00 & $2015 / 02 / 25$ & 200 & 1 & $3.796 \pm 0.055$ & $-0.269 \pm 0.006$ \\
\hline HD 65583 & $R$ & 6.4 & $2015 / 02 / 25$ & 200 & 1 & $3.623 \pm 0.042$ & $-0.225 \pm 0.007$ \\
\hline HD 90508 & $R$ & 5.98 & $2015 / 02 / 18$ & 20 & 0.5 & $3.612 \pm 0.022$ & $-0.325 \pm 0.015$ \\
\hline HD 90508 & V & 6.45 & $2015 / 02 / 22$ & 20 & 0.5 & $3.845 \pm 0.018$ & $-0.225 \pm 0.025$ \\
\hline HD 90508 & $R$ & 5.98 & $2015 / 02 / 22$ & 20 & 0.5 & $3.591 \pm 0.016$ & $-0.171 \pm 0.015$ \\
\hline HD 90508 & $V$ & 6.45 & $2015 / 03 / 03$ & 650 & 1 & $3.849 \pm 0.051$ & $-0.272 \pm 0.012$ \\
\hline HD 90508 & $V$ & 6.45 & $2015 / 03 / 05$ & 4800 & 1 & $3.836 \pm 0.011$ & $-0.235 \pm 0.010$ \\
\hline HD 90508 & $V$ & 6.45 & $2015 / 03 / 07$ & 2500 & 1 & $3.775 \pm 0.014$ & $-0.285 \pm 0.010$ \\
\hline HD 90508 & $V$ & 6.45 & $2015 / 03 / 28$ & 50 & 1.5 & $3.827 \pm 0.008$ & $-0.258 \pm 0.007$ \\
\hline HD 90508 & $V$ & 6.45 & $2015 / 03 / 30$ & 1100 & 1 & $3.857 \pm 0.023$ & $-0.308 \pm 0.002$ \\
\hline HD 90508 & $V$ & 6.45 & $2015 / 12 / 10$ & 20 & 1 & $3.754 \pm 0.014$ & $-0.747 \pm 0.037$ \\
\hline
\end{tabular}


Table A1 - continued

\begin{tabular}{|c|c|c|c|c|c|c|c|}
\hline Star & Filter & Mag & Obs date & $N_{\text {obs }}$ & $\begin{array}{l}t_{\exp } \\
(\mathrm{s})\end{array}$ & $\begin{array}{c}q_{0} \\
\left(\times 10^{-2}\right)\end{array}$ & $\begin{array}{c}u_{0} \\
\left(\times 10^{-2}\right)\end{array}$ \\
\hline HD 90508 & V & 6.45 & 2015/12/11 & 32 & 1 & $3.787 \pm 0.014$ & $-0.775 \pm 0.026$ \\
\hline HD 98281 & $V$ & 7.23 & $2015 / 02 / 22$ & 20 & 2 & $3.828 \pm 0.014$ & $-0.240 \pm 0.017$ \\
\hline HD 98281 & $R$ & 6.82 & $2015 / 02 / 22$ & 20 & 2 & $3.640 \pm 0.009$ & $-0.190 \pm 0.013$ \\
\hline HD 103095 & $V$ & 6.45 & $2015 / 02 / 22$ & 24 & 0.5 & $3.788 \pm 0.023$ & $-0.224 \pm 0.011$ \\
\hline HD 103095 & $R$ & 5.80 & $2015 / 02 / 22$ & 20 & 0.5 & $3.645 \pm 0.082$ & $-0.284 \pm 0.015$ \\
\hline HD 103095 & V & 6.45 & $2015 / 03 / 27$ & 150 & 2 & $3.821 \pm 0.011$ & $-0.338 \pm 0.009$ \\
\hline HD 103095 & $V$ & 6.45 & $2015 / 03 / 28$ & 230 & 1.5 & $3.835 \pm 0.073$ & $-0.306 \pm 0.006$ \\
\hline HD 114710 & V & 3.25 & $2015 / 02 / 22$ & 20 & 0.1 & $3.850 \pm 0.011$ & $-0.534 \pm 0.022$ \\
\hline HD 114710 & $R$ & 3.77 & $2015 / 02 / 22$ & 20 & 0.1 & $3.606 \pm 0.015$ & $-0.423 \pm 0.011$ \\
\hline HD 114710 & V & 3.25 & $2015 / 02 / 25$ & 100 & 0.1 & $3.835 \pm 0.036$ & $-0.486 \pm 0.011$ \\
\hline HD 114710 & $R$ & 3.77 & $2015 / 02 / 25$ & 100 & 0.1 & $3.604 \pm 0.007$ & $-0.333 \pm 0.009$ \\
\hline HD 144287 & $V$ & 7.06 & $2015 / 02 / 18$ & 19 & 1 & $3.757 \pm 0.016$ & $-0.325 \pm 0.012$ \\
\hline HD 144287 & $R$ & 7.06 & $2015 / 02 / 18$ & 22 & 1 & $3.656 \pm 0.016$ & $-0.307 \pm 0.013$ \\
\hline HD 144287 & $V$ & 7.06 & $2015 / 02 / 22$ & 20 & 1 & $3.580 \pm 0.026$ & $-0.265 \pm 0.014$ \\
\hline HD 144287 & $R$ & 7.06 & $2015 / 02 / 22$ & 20 & 1 & $3.477 \pm 0.022$ & $-0.382 \pm 0.027$ \\
\hline HD 144287 & $V$ & 7.06 & $2015 / 02 / 25$ & 100 & 1 & $3.797 \pm 0.008$ & $-0.256 \pm 0.008$ \\
\hline HD 142373 & V & 4.62 & $2015 / 02 / 22$ & 21 & 0.1 & $4.000 \pm 0.014$ & $-0.432 \pm 0.022$ \\
\hline HD 142373 & $R$ & 4.14 & $2015 / 02 / 22$ & 20 & 0.1 & $3.732 \pm 0.018$ & $-0.460 \pm 0.015$ \\
\hline HD 142373 & $V$ & 4.62 & $2015 / 02 / 25$ & 100 & 0.5 & $3.823 \pm 0.036$ & $-0.247 \pm 0.005$ \\
\hline HD 144579 & $V$ & 6.67 & $2015 / 02 / 22$ & 20 & 0.5 & $3.837 \pm 0.022$ & $-0.285 \pm 0.023$ \\
\hline HD 144579 & $R$ & 6.04 & $2015 / 02 / 22$ & 20 & 0.5 & $3.636 \pm 0.020$ & $-0.300 \pm 0.020$ \\
\hline HD 154345 & V & 6.74 & $2015 / 07 / 17$ & 30 & 1 & $3.876 \pm 0.012$ & $-0.322 \pm 0.016$ \\
\hline HD 154345 & $V$ & 6.74 & $2015 / 07 / 23$ & 30 & 1 & $3.776 \pm 0.020$ & $-0.336 \pm 0.017$ \\
\hline HD 154345 & V & 6.74 & $2015 / 07 / 17$ & 30 & 1 & $3.876 \pm 0.012$ & $-0.322 \pm 0.016$ \\
\hline HD 154345 & $V$ & 6.74 & $2015 / 07 / 23$ & 30 & 1 & $3.776 \pm 0.020$ & $-0.336 \pm 0.017$ \\
\hline HD 165908 & $V$ & 5.07 & $2015 / 07 / 17$ & 30 & 0.1 & $3.920 \pm 0.014$ & $-0.356 \pm 0.014$ \\
\hline HD 165908 & $V$ & 5.07 & $2015 / 07 / 18$ & 30 & 0.1 & $3.960 \pm 0.054$ & $-0.337 \pm 0.029$ \\
\hline HD 165908 & $V$ & 5.07 & $2015 / 07 / 23$ & 30 & 0.1 & $3.881 \pm 0.017$ & $-0.550 \pm 0.016$ \\
\hline HD 185395 & $V$ & 4.48 & $2015 / 07 / 13$ & 30 & 0.1 & $3.892 \pm 0.026$ & $-0.584 \pm 0.032$ \\
\hline HD 185395 & V & 4.48 & $2015 / 07 / 14$ & 30 & 0.1 & $3.861 \pm 0.023$ & $-0.587 \pm 0.022$ \\
\hline HD 185395 & $V$ & 4.48 & $2015 / 07 / 15$ & 30 & 0.1 & $3.860 \pm 0.034$ & $-0.478 \pm 0.064$ \\
\hline HD 185395 & $V$ & 4.48 & $2015 / 07 / 18$ & 30 & 0.1 & $3.935 \pm 0.022$ & $-0.432 \pm 0.067$ \\
\hline HD 185395 & $V$ & 4.48 & $2015 / 07 / 21$ & 30 & 0.1 & $3.967 \pm 0.050$ & $-0.483 \pm 0.022$ \\
\hline HD 188512 & $V$ & 3.71 & $2015 / 07 / 21$ & 30 & 0.03 & $3.883 \pm 0.012$ & $-0.473 \pm 0.002$ \\
\hline HD 202573 & $V$ & 6.98 & $2015 / 07 / 16$ & 30 & 1 & $3.783 \pm 0.038$ & $-0.334 \pm 0.002$ \\
\hline HD 202573 & V & 6.98 & $2015 / 11 / 15$ & 30 & 2.5 & $3.722 \pm 0.079$ & $-0.466 \pm 0.008$ \\
\hline HD 202573 & V & 6.98 & $2015 / 12 / 08$ & 31 & 0.5 & $3.814 \pm 0.017$ & $-0.558 \pm 0.023$ \\
\hline HD 202573 & V & 6.98 & $2015 / 12 / 10$ & 30 & 1 & $3.760 \pm 0.015$ & $-0.583 \pm 0.014$ \\
\hline HD 202573 & $V$ & 6.98 & $2015 / 12 / 11$ & 30 & 1 & $3.671 \pm 0.019$ & $-0.609 \pm 0.018$ \\
\hline HD 210027 & V & 3.77 & $2015 / 07 / 16$ & 30 & 0.05 & $4.009 \pm 0.035$ & $-0.504 \pm 0.001$ \\
\hline
\end{tabular}


Table A2. Summary table of all observations for polarized standard stars. The first column corresponds to the name of the star. The third column reports the magnitude in the respective filter (second column). $N_{\text {obs }}$ represents the number of observed frames, $t_{\exp }$ is the exposure time of the individual frames, $P$ is the measured linear polarization (after correction for the instrumental polarization), $\mathrm{O}-\mathrm{C}(P)$ is the difference between the observed and the expected linear polarization from reference sources, $\theta$ is the measured polarization angle with respect to the north direction and $\mathrm{O}-\mathrm{C}(\theta)$ is the difference between the observed and the expected polarization angles from reference sources.

\begin{tabular}{lccccccrrr}
\hline Star & Filter & Mag & Obs date & $N_{\text {obs }}$ & $\begin{array}{c}t_{\text {exp }} \\
(\mathrm{s})\end{array}$ & $\begin{array}{c}P \\
\left(\times 10^{-2}\right)\end{array}$ & $\begin{array}{r}\mathrm{O}-\mathrm{C}(P) \\
\left(\times 10^{-2}\right)\end{array}$ & $\begin{array}{c}\theta \\
(\mathrm{deg})\end{array}$ & $\begin{array}{c}\mathrm{O}-\mathrm{C}(\theta) \\
(\mathrm{deg})\end{array}$ \\
\hline HD 14433 & $V$ & 6.39 & $2015 / 02 / 19$ & 23 & 1 & $3.82 \pm 0.10$ & -0.05 & $111.4 \pm 0.7$ & -1.1 \\
HD 14433 & $R$ & 5.79 & $2015 / 02 / 19$ & 23 & 1 & $3.66 \pm 0.09$ & -0.03 & $111.7 \pm 0.6$ & -0.3 \\
HD 14433 & $V$ & 6.39 & $2015 / 02 / 22$ & 20 & 1 & $4.03 \pm 0.02$ & 0.15 & $112.8 \pm 0.2$ & 0.3 \\
HD 14433 & $R$ & 5.79 & $2015 / 02 / 22$ & 22 & 1 & $3.72 \pm 0.03$ & 0.03 & $113.1 \pm 0.2$ & 1 \\
HD 23512 & $V$ & 8.09 & $2015 / 02 / 19$ & 20 & 5 & $2.29 \pm 0.10$ & 0.08 & $27.5 \pm 0.7$ & -0.6 \\
HD 23512 & $R$ & 7.79 & $2015 / 02 / 19$ & 20 & 5 & $2.14 \pm 0.09$ & 0.06 & $27.6 \pm 0.6$ & -2 \\
HD 43384 & $V$ & 6.25 & $2015 / 02 / 18$ & 60 & 0.5 & $2.97 \pm 0.04$ & 0.12 & $170.0 \pm 0.3$ & 2.6 \\
HD 43384 & $R$ & 5.78 & $2015 / 02 / 18$ & 60 & 0.5 & $2.89 \pm 0.04$ & 0.09 & $170.3 \pm 0.3$ & 0.5 \\
HD 43384 & $V$ & 6.25 & $2015 / 10 / 16$ & 60 & 0.5 & $2.75 \pm 0.02$ & -0.10 & $171.0 \pm 0.2$ & 3.6 \\
HD 43384 & $V$ & 6.25 & $2015 / 12 / 09$ & 60 & 0.5 & $2.85 \pm 0.04$ & 0.00 & $169.7 \pm 0.3$ & 1.3 \\
HD 43384 & $V$ & 6.25 & $2015 / 12 / 11$ & 60 & 0.5 & $2.89 \pm 0.05$ & 0.04 & $170.0 \pm 0.4$ & 2.6 \\
HD 154445 & $V$ & 5.61 & $2015 / 02 / 18$ & 20 & 0.2 & $3.67 \pm 0.05$ & 0.03 & $89.1 \pm 0.2$ & -0.6 \\
HD 154445 & $R$ & 5.39 & $2015 / 02 / 18$ & 20 & 0.2 & $3.72 \pm 0.05$ & 0.29 & $88.9 \pm 0.3$ & 0.2 \\
HD 154445 & $V$ & 5.61 & $2015 / 02 / 25$ & 20 & 1 & $3.74 \pm 0.10$ & 0.02 & $89.0 \pm 0.2$ & -0.7 \\
HD 154445 & $V$ & 5.61 & $2015 / 03 / 27$ & 40 & 1 & $3.66 \pm 0.03$ & -0.06 & $88.5 \pm 0.2$ & -1.2 \\
HD 154445 & $V$ & 5.61 & $2015 / 05 / 12$ & 30 & 1 & $3.72 \pm 0.02$ & 0.00 & $88.8 \pm 0.2$ & -0.7 \\
HD 154445 & $V$ & 5.61 & $2015 / 07 / 23$ & 30 & 1 & $3.68 \pm 0.06$ & -0.04 & $88.3 \pm 0.5$ & -1.4 \\
HD 183143 & $V$ & 6.86 & $2015 / 07 / 14$ & 30 & 1 & $6.03 \pm 0.06$ & 0.09 & $0.2 \pm 0.3$ & 0.3 \\
HD 183143 & $V$ & 6.86 & $2015 / 07 / 17$ & 30 & 1 & $5.94 \pm 0.03$ & 0.00 & $178.8 \pm 0.2$ & -1.1 \\
HD 183143 & $V$ & 6.86 & $2015 / 07 / 18$ & 30 & 1 & $5.92 \pm 0.04$ & -0.02 & $179.3 \pm 0.2$ & -0.6 \\
HD 187929 & $V$ & 3.80 & $2015 / 07 / 13$ & 30 & 0.1 & $1.77 \pm 0.04$ & 0.01 & $92.3 \pm 0.5$ & 0.3 \\
HD 187929 & $V$ & 3.80 & $2015 / 07 / 15$ & 30 & 0.1 & $1.63 \pm 0.04$ & -0.13 & $92.8 \pm 0.4$ & 0.8 \\
HD 198478 & $V$ & 4.86 & $2015 / 07 / 21$ & 30 & 0.5 & $2.68 \pm 0.05$ & -0.01 & $2.0 \pm 0.7$ & -3.1 \\
HD 204827 & $V$ & 7.94 & $2015 / 07 / 16$ & 30 & 2 & $5.62 \pm 0.06$ & 0.21 & $58.7 \pm 0.3$ & -0.3 \\
HD 251204 & $V$ & 10.2 & $2015 / 02 / 18$ & 20 & 10 & $4.98 \pm 0.03$ & 0.63 & $163.2 \pm 0.3$ & 12.1 \\
\hline & & & & & & & & & 0.3 \\
\hline
\end{tabular}

Table A3. References for the polarized standard stars which were observed. The degree of polarization and polarization angle correspond to the mean value of all reported values in the literature.

\begin{tabular}{|c|c|c|c|c|c|}
\hline Star & Filter & Mag & $\begin{array}{c}P \\
\left(\times 10^{-2}\right)\end{array}$ & $\begin{array}{c}\theta \\
(\operatorname{deg})\end{array}$ & References \\
\hline HD 14433 & $V$ & 6.39 & $3.87 \pm 0.01$ & $112.5 \pm 0.1$ & Hsu \& Breger (1982) \\
\hline HD 23512 & $V$ & 8.09 & $2.21 \pm 0.02$ & $28.1 \pm 1.2$ & Hsu \& Breger (1982); Bastien et al. (1988) \\
\hline HD 23512 & $R$ & 7.79 & $2.20 \pm 0.02$ & $29.6 \pm 1.2$ & Hsu \& Breger (1982) \\
\hline HD 43384 & $V$ & 6.25 & $2.85 \pm 0.10$ & $168.4 \pm 1.4$ & Hsu \& Breger (1982) \\
\hline HD 43384 & $R$ & 5.78 & $2.80 \pm 0.03$ & $169.8 \pm 0.9$ & Hsu \& Breger (1982) \\
\hline HD 154445 & $R$ & 5.39 & $3.43 \pm 0.16$ & $89.7 \pm 0.4$ & Hsu \& Breger (1982); Schmidt et al. (1992); Wolff, Nordsieck \& Nook (1996) \\
\hline HD 183143 & $V$ & 6.86 & $5.94 \pm 0.13$ & 179.9 & $\begin{array}{c}\text { Schulz \& Lenzen (1983); Bailey \& Hough (1982); } \\
\text { Clemens \& Tapia (1990) }\end{array}$ \\
\hline HD 187929 & $V$ & 3.80 & $1.76 \pm 0.04$ & $92.0 \pm 0.7$ & Bastien et al. (1988); Bailey \& Hough (1982) \\
\hline HD 198478 & $V$ & 4.86 & $2.69 \pm 0.07$ & 5.1 & Hsu \& Breger (1982) \\
\hline
\end{tabular}




\section{APPENDIX B: SUMMARY OF CURRENTLY AVAILABLE CAPS DATA}

Table B1. Summary table of all the asteroids observed by the CAPS. The first column gives the number of the asteroid and the second column gives the date of observation. $\alpha$ is the phase angle during the observation, $P_{\mathrm{r}}$ is the linear polarization degree and the last column shows the filter used.

\begin{tabular}{|c|c|c|c|c|}
\hline Object no. & Date & $\begin{array}{c}\alpha \\
(\operatorname{deg})\end{array}$ & $\begin{array}{c}P_{\mathrm{r}} \\
\text { (per cent) }\end{array}$ & Filter \\
\hline 2 & $2015 / 02 / 18$ & 18.70 & $0.052 \pm 0.032$ & $V$ \\
\hline 2 & $2015 / 02 / 18$ & 18.70 & $0.081 \pm 0.039$ & $R$ \\
\hline 3 & $2015 / 02 / 18$ & 11.91 & $-0.658 \pm 0.022$ & $V$ \\
\hline 3 & $2015 / 02 / 18$ & 11.91 & $-0.645 \pm 0.019$ & $R$ \\
\hline 6 & $2015 / 02 / 19$ & 27.14 & $0.463 \pm 0.035$ & $V$ \\
\hline 6 & $2015 / 02 / 19$ & 27.14 & $0.453 \pm 0.038$ & $R$ \\
\hline 7 & $2015 / 02 / 18$ & 8.24 & $-0.635 \pm 0.026$ & $V$ \\
\hline 7 & $2015 / 02 / 18$ & 8.24 & $-0.825 \pm 0.030$ & $R$ \\
\hline 8 & $2015 / 02 / 18$ & 3.33 & $-0.468 \pm 0.025$ & $V$ \\
\hline 8 & $2015 / 02 / 18$ & 3.33 & $-0.593 \pm 0.022$ & $R$ \\
\hline 8 & $2015 / 03 / 11$ & 12.40 & $-0.320 \pm 0.025$ & $V$ \\
\hline 10 & $2015 / 02 / 18$ & 14.45 & $-0.790 \pm 0.030$ & $V$ \\
\hline 10 & 2015/02/18 & 14.45 & $-0.776 \pm 0.045$ & $R$ \\
\hline 11 & $2015 / 02 / 18$ & 21.08 & $0.251 \pm 0.028$ & $V$ \\
\hline 11 & $2015 / 02 / 18$ & 21.08 & $0.151 \pm 0.025$ & $R$ \\
\hline 11 & $2015 / 05 / 20$ & 12.75 & $-0.642 \pm 0.024$ & $V$ \\
\hline 15 & $2015 / 07 / 15$ & 26.05 & $0.391 \pm 0.054$ & $V$ \\
\hline 17 & $2015 / 02 / 18$ & 8.14 & $-0.710 \pm 0.033$ & $V$ \\
\hline 17 & $2015 / 02 / 18$ & 8.14 & $-0.707 \pm 0.024$ & $R$ \\
\hline 18 & $2015 / 02 / 19$ & 20.25 & $-0.283 \pm 0.047$ & $V$ \\
\hline 18 & 2015/02/19 & 20.25 & $0.244 \pm 0.052$ & $R$ \\
\hline 20 & 2015/02/19 & 22.38 & $0.247 \pm 0.048$ & $V$ \\
\hline 20 & 2015/02/19 & 22.38 & $0.176 \pm 0.043$ & $R$ \\
\hline 23 & 2015/02/19 & 28.37 & $0.616 \pm 0.031$ & $V$ \\
\hline 23 & 2015/02/19 & 28.37 & $0.757 \pm 0.034$ & $R$ \\
\hline 25 & $2015 / 02 / 22$ & 20.15 & $0.157 \pm 0.039$ & $R$ \\
\hline 26 & $2015 / 10 / 15$ & 14.00 & $-0.515 \pm 0.023$ & $V$ \\
\hline 29 & 2015/10/15 & 5.33 & $-0.802 \pm 0.010$ & $V$ \\
\hline 35 & 2015/02/18 & 16.96 & $-1.170 \pm 0.069$ & $V$ \\
\hline 38 & $2015 / 02 / 18$ & 2.74 & $-1.016 \pm 0.033$ & $V$ \\
\hline 38 & $2015 / 02 / 18$ & 2.74 & $-0.998 \pm 0.038$ & $R$ \\
\hline 42 & $2015 / 02 / 18$ & 8.09 & $-0.638 \pm 0.029$ & $V$ \\
\hline 42 & $2015 / 02 / 18$ & 8.09 & $-0.679 \pm 0.034$ & $R$ \\
\hline 43 & 2015/10/15 & 16.10 & $-0.270 \pm 0.022$ & $V$ \\
\hline 44 & $2015 / 02 / 18$ & 17.17 & $-0.033 \pm 0.031$ & $V$ \\
\hline 44 & $2015 / 02 / 18$ & 17.17 & $-0.020 \pm 0.025$ & $R$ \\
\hline 44 & $2015 / 05 / 13$ & 21.53 & $0.063 \pm 0.032$ & $V$ \\
\hline 45 & $2015 / 07 / 15$ & 19.84 & $1.009 \pm 0.105$ & $V$ \\
\hline 45 & 2015/07/17 & 19.54 & $0.983 \pm 0.029$ & $V$ \\
\hline 48 & $2015 / 07 / 13$ & 12.83 & $-2.035 \pm 0.052$ & $V$ \\
\hline 48 & 2015/07/14 & 13.04 & $-1.923 \pm 0.053$ & $V$ \\
\hline 50 & $2015 / 02 / 18$ & 12.96 & $-1.656 \pm 0.051$ & $V$ \\
\hline 51 & $2015 / 05 / 12$ & 14.72 & $-1.342 \pm 0.021$ & $V$ \\
\hline 51 & $2015 / 07 / 14$ & 17.12 & $-0.673 \pm 0.060$ & $V$ \\
\hline 51 & $2015 / 10 / 14$ & 22.64 & $0.439 \pm 0.052$ & $V$ \\
\hline 53 & 2015/02/19 & 19.32 & $-0.298 \pm 0.050$ & $V$ \\
\hline 53 & 2015/02/19 & 19.32 & $-0.369 \pm 0.045$ & $R$ \\
\hline 56 & 2015/02/19 & 6.39 & $-0.918 \pm 0.068$ & $V$ \\
\hline 56 & $2015 / 02 / 19$ & 6.39 & $-1.128 \pm 0.045$ & $R$ \\
\hline 57 & $2015 / 07 / 14$ & 7.01 & $-0.758 \pm 0.057$ & $V$ \\
\hline 57 & $2015 / 07 / 15$ & 6.90 & $-0.801 \pm 0.047$ & $V$ \\
\hline 58 & $2015 / 02 / 22$ & 6.05 & $-0.861 \pm 0.022$ & $V$ \\
\hline 59 & $2015 / 05 / 12$ & 7.67 & $-1.134 \pm 0.027$ & $V$ \\
\hline 61 & $2015 / 07 / 22$ & 23.83 & $0.327 \pm 0.047$ & $V$ \\
\hline 69 & $2015 / 02 / 19$ & 15.64 & $-0.795 \pm 0.026$ & $V$ \\
\hline 69 & $2015 / 02 / 19$ & 15.64 & $-0.769 \pm 0.034$ & $R$ \\
\hline 69 & $2015 / 05 / 21$ & 21.95 & $0.242 \pm 0.023$ & $V$ \\
\hline 71 & 2015/02/19 & 7.64 & $-0.500 \pm 0.031$ & $V$ \\
\hline 71 & $2015 / 02 / 19$ & 7.64 & $-0.420 \pm 0.047$ & $R$ \\
\hline 76 & $2015 / 02 / 19$ & 3.23 & $-0.545 \pm 0.045$ & $V$ \\
\hline
\end{tabular}

Table B1 - continued

\begin{tabular}{|c|c|c|c|c|}
\hline Object no. & Date & $\begin{array}{c}\alpha \\
(\operatorname{deg})\end{array}$ & $\begin{array}{c}P_{\mathrm{r}} \\
\text { (per cent) }\end{array}$ & Filter \\
\hline 76 & $2015 / 02 / 19$ & 3.23 & $-0.664 \pm 0.034$ & $R$ \\
\hline 78 & 2015/03/07 & 24.82 & $0.940 \pm 0.056$ & $V$ \\
\hline 86 & 2015/02/19 & 6.27 & $-1.389 \pm 0.076$ & $V$ \\
\hline 86 & 2015/02/19 & 6.27 & $-1.239 \pm 0.053$ & $R$ \\
\hline 89 & $2015 / 02 / 19$ & 6.09 & $-0.808 \pm 0.025$ & $V$ \\
\hline 89 & 2015/02/19 & 6.09 & $-0.821 \pm 0.034$ & $R$ \\
\hline 94 & $2015 / 02 / 22$ & 16.63 & $-0.397 \pm 0.034$ & $V$ \\
\hline 96 & $2015 / 07 / 21$ & 17.22 & $-0.916 \pm 0.055$ & $V$ \\
\hline 96 & $2015 / 07 / 23$ & 17.22 & $-0.841 \pm 0.055$ & $V$ \\
\hline 103 & 2015/10/10 & 13.70 & $-0.494 \pm 0.026$ & $V$ \\
\hline 122 & $2015 / 05 / 20$ & 3.07 & $-0.453 \pm 0.037$ & $V$ \\
\hline 122 & $2015 / 05 / 22$ & 3.45 & $-0.513 \pm 0.031$ & $V$ \\
\hline 126 & $2015 / 02 / 22$ & 4.20 & $-0.690 \pm 0.025$ & $V$ \\
\hline 128 & $2015 / 02 / 22$ & 9.27 & $-1.507 \pm 0.019$ & $R$ \\
\hline 133 & $2015 / 02 / 22$ & 15.78 & $-0.468 \pm 0.069$ & $R$ \\
\hline 139 & $2015 / 03 / 28$ & 23.05 & $0.810 \pm 0.029$ & $V$ \\
\hline 165 & 2015/07/16 & 19.59 & $0.078 \pm 0.060$ & $V$ \\
\hline 168 & $2015 / 02 / 22$ & 12.94 & $-1.752 \pm 0.036$ & $R$ \\
\hline 185 & $2015 / 07 / 16$ & 18.56 & $-0.008 \pm 0.056$ & $V$ \\
\hline 191 & 2015/07/16 & 12.89 & $-0.902 \pm 0.056$ & $V$ \\
\hline 192 & 2015/07/16 & 33.18 & $1.023 \pm 0.050$ & $V$ \\
\hline 194 & $2015 / 05 / 21$ & 12.40 & $-1.692 \pm 0.025$ & $V$ \\
\hline 194 & 2015/07/17 & 24.72 & $1.295 \pm 0.036$ & $V$ \\
\hline 200 & $2015 / 07 / 22$ & 13.40 & $-1.725 \pm 0.043$ & $V$ \\
\hline 200 & $2015 / 10 / 15$ & 19.25 & $-0.596 \pm 0.022$ & $V$ \\
\hline 201 & 2015/07/14 & 23.55 & $0.276 \pm 0.058$ & $V$ \\
\hline 201 & $2015 / 07 / 15$ & 23.35 & $0.183 \pm 0.056$ & $V$ \\
\hline 213 & 2015/10/16 & 17.30 & $-0.352 \pm 0.026$ & $V$ \\
\hline 221 & 2015/07/14 & 17.78 & $-0.587 \pm 0.056$ & $V$ \\
\hline 225 & 2015/10/16 & 13.83 & $-0.735 \pm 0.024$ & $V$ \\
\hline 234 & $2015 / 02 / 25$ & 16.95 & $-1.512 \pm 0.047$ & $V$ \\
\hline 234 & $2015 / 05 / 22$ & 17.38 & $-1.424 \pm 0.023$ & $V$ \\
\hline 236 & $2015 / 02 / 22$ & 11.50 & $-1.285 \pm 0.065$ & $V$ \\
\hline 236 & $2015 / 03 / 30$ & 0.41 & $0.021 \pm 0.035$ & $V$ \\
\hline 236 & $2015 / 05 / 21$ & 15.26 & $-1.232 \pm 0.045$ & $V$ \\
\hline 273 & $2015 / 07 / 23$ & 17.87 & $-0.345 \pm 0.058$ & $V$ \\
\hline 276 & $2015 / 07 / 23$ & 15.74 & $-0.305 \pm 0.045$ & $V$ \\
\hline 304 & $2015 / 07 / 17$ & 19.89 & $0.305 \pm 0.045$ & $V$ \\
\hline 306 & 2015/07/14 & 4.59 & $-0.354 \pm 0.063$ & $V$ \\
\hline 313 & 2015/07/14 & 6.67 & $-1.835 \pm 0.052$ & $V$ \\
\hline 313 & $2015 / 07 / 18$ & 5.89 & $-1.502 \pm 0.032$ & $V$ \\
\hline 324 & $2015 / 03 / 27$ & 11.99 & $-1.409 \pm 0.057$ & $V$ \\
\hline 337 & $2015 / 02 / 25$ & 18.22 & $-0.324 \pm 0.017$ & $R$ \\
\hline 426 & $2015 / 07 / 17$ & 17.27 & $-0.427 \pm 0.055$ & $V$ \\
\hline 426 & $2015 / 07 / 18$ & 17.20 & $-0.579 \pm 0.087$ & $V$ \\
\hline 444 & $2015 / 05 / 21$ & 6.55 & $-1.153 \pm 0.023$ & $V$ \\
\hline 444 & $2015 / 07 / 13$ & 15.80 & $-0.624 \pm 0.054$ & $V$ \\
\hline 444 & $2015 / 07 / 17$ & 17.42 & $-0.615 \pm 0.047$ & $V$ \\
\hline 478 & $2015 / 07 / 18$ & 7.80 & $-0.735 \pm 0.056$ & $V$ \\
\hline 478 & $2015 / 07 / 21$ & 8.60 & $-0.744 \pm 0.048$ & $V$ \\
\hline 478 & $2015 / 09 / 29$ & 17.82 & $-0.100 \pm 0.100$ & $V$ \\
\hline 491 & $2015 / 07 / 21$ & 11.93 & $-0.963 \pm 0.075$ & $V$ \\
\hline 500 & 2015/07/17 & 7.91 & $-0.585 \pm 0.033$ & $V$ \\
\hline 511 & $2015 / 02 / 25$ & 9.78 & $-1.654 \pm 0.023$ & $V$ \\
\hline 532 & $2015 / 07 / 18$ & 21.44 & $-0.007 \pm 0.038$ & $V$ \\
\hline 679 & $2015 / 07 / 13$ & 3.72 & $-0.522 \pm 0.059$ & $V$ \\
\hline 690 & $2015 / 07 / 24$ & 21.70 & $0.559 \pm 0.100$ & $V$ \\
\hline 783 & $2015 / 07 / 21$ & 15.40 & $-1.319 \pm 0.047$ & $V$ \\
\hline 824 & $2015 / 07 / 13$ & 3.35 & $-0.735 \pm 0.138$ & $V$ \\
\hline 824 & $2015 / 07 / 15$ & 3.74 & $-0.904 \pm 0.064$ & $V$ \\
\hline 849 & 2015/07/17 & 13.19 & $-0.713 \pm 0.046$ & $V$ \\
\hline 849 & 2015/07/18 & 13.04 & $-0.755 \pm 0.045$ & $V$ \\
\hline 1372 & $2015 / 02 / 26$ & 19.96 & $-0.713 \pm 0.218$ & $V$ \\
\hline 1702 & $2015 / 05 / 22$ & 7.39 & $-1.136 \pm 0.550$ & $V$ \\
\hline
\end{tabular}

